\title{
An Efficient Algorithm for Guard Placement in Polygons with Holes*
}

\author{
I. Bjorling-Sachs ${ }^{1}$ and D. L. Souvaine ${ }^{2}$ \\ ${ }^{1}$ Department of Computer Science, Lafayette College, \\ Easton, PA 18042, USA \\ bjorling@lafcol.lafayette.edu \\ ${ }^{2}$ Department of Computer Science, Rutgers University, \\ New Brunswick, NJ 08903, USA \\ dls@,cs.rutgers.edu
}

\begin{abstract}
In this paper we consider the problem of placing guards to supervise an art gallery with holes. No gallery with $n$ vertices and $h$ holes requires more than $L(n+h) / 3\rfloor$ guards. For some galleries this number of guards is necessary. We present an algorithm which places the $\lfloor(n+h) / 3\rfloor$ guards in $O\left(n^{2}\right)$ time.
\end{abstract}

\section{Introduction}

Art-gallery problems ask the following question: How many guards are always sufficient and sometimes necessary to see every point in an art gallery? The floor plan of the gallery is viewed as a simple polygon. A guard is a point in the polygon. If this point coincides with vertex, then the guard is called a vertex guard. If there is no restriction on the location of the point, then the guard is a point guard. We are concerned with the visibility between the guards and the remaining points in the polygon. A guard $x$ sees a point $y$ if the line segment $\overline{x y}$ does not intersect the exterior of the polygon. The original art-gallery problem, posed in 1973 by Klee [7], asks for the number of guards required to survey any art gallery with $n$ walls and no interior obstructions. The problem was soon solved by Chvátal [3], who showed that $\lfloor n / 3\rfloor$ vertex guards are both necessary and sufficient. A different and very simple sufficiency proof of the same result was given in 1978

* Work by the first author was done at Rutgers University and was supported in part by a DIMACS research assistantship. The second author was supported in part by National Science Foundation Grants CCR-88-03549 and CCR-91-04732. 
by Fisk [4]. This proof was used by Avis and Toussaint to implement an algorithm running in $O(n \log n)$ time, to assign positions to the guards [1].

If the art gallery is allowed to have obstructions (e.g., pillars) in its interior, the corresponding floor plan is a simple polygon with other simple disjoint polygons called holes inside. In such a polygon, $\lfloor n / 3\rfloor$ guards are no longer sufficient. In 1984 Shermer showed that, for any polygon with one hole, $\lfloor(n+1) / 3\rfloor$ is a tight bound on the number of guards needed. Shermer had already established in 1982 that $\lfloor(n+h) / 3\rfloor$ guards are necessary for a polygon with $n$ vertices and $h$ holes. Shermer's results can be found in [8]. In 1991 two independent and dramatically different proofs were generated for the fact that $\lfloor(n+h) / 3\rfloor$ point guards are also sufficient: one by us [2] and one by Hoffmann et al. [6]. Neither paper gave details of algorithms for placing the guards, but the algorithm derived from the Hoffmann et al. proof has complexity $O\left(n^{3} \log n\right)$ [5]. In this paper we present an $O\left(n^{2}\right)$ algorithm to place the guards. Since the algorithm derives from the constructive nature of our proof, we start by giving the proof.

Fisk's proof that $\lfloor n / 3\rfloor$ guards are sufficient in any polygon without holes, starts with an arbitrary triangulation of the polygon. The second step is to three-color the graph of the triangulated polygon, i.e., colors are assigned to the vertices in such a way that no two vertices that share an edge in the graph have the same color and only three colors are used. In the third step guards are placed at the vertices of the least-used color. At most $\lfloor n / 3\rfloor$ vertices can be colored by this color, so no more than $\lfloor n / 3\rfloor$ guards are used. Finally it remains to note that since all three vertices of a triangle have different colors, every triangle has a guard at one of its vertices. This guard sees every point inside the triangle. Every point of the polygon is inside some triangle and thus the guards see all points in the polygon. To show the sufficiency of $\lfloor(n+1) / 3\rfloor$ guards in any polygon with exactly one hole, Shermer uses an arbitrary triangulation of the polygon with the hole. This triangulation must contain a cycle of triangles, i.e., if we construct the dual of the triangulation with one node for each triangle and an edge between two nodes if the corresponding triangles share an edge, then the dual graph has a cycle surrounding the hole. Shermer first shows that to prove the sufficiency of $\lfloor(n+1) / 3\rfloor$ guards for any triangulation, it is enough to provide a proof for a reduced triangulation consisting only of a cycle of triangles perhaps with at most single triangles attached. In some of these triangulations it is not possible to pick $\lfloor(n+1) / 3\rfloor$ guards so that every triangle has a guard at one of its vertices. Shermer calls these configurations tough triangulations and makes a case study to show that in each case $\lfloor(n+1) / 3\rfloor$ guards are still sufficient.

Our approach to show that $\lfloor(n+h) / 3\rfloor$ guards are sufficient in any polygon with $n$ vertices and $h$ holes, is radically different: first connect each hole to the exterior with a quadrilateral "channel" and then triangulate the hole-free version of the polygon. The channels are such that:

(1) Only one new vertex is introduced for each channel.

(2) A triangle in the remaining polygon sees all of the channel.

Our triangulation is not arbitrary. We force these special triangles to be included with the result that a guard placement based on three-coloring in the hole-free 
polygon will automatically cover the channels. In Section 2 we use this method to give a new proof for the sufficiency of $\lfloor(n+1) / 3\rfloor$ guards when $h=1$ and in Section 3 we extend this result to an arbitrary $h$. The computational complexity and other aspects of the algorithm is discussed in Section 4.

\section{Guarding a Polygon with One Hole}

Let $P$ be a polygon with one hole and $n$ vertices. Our procedure for proving that $P$ can be guarded by $\lfloor(n+1) / 3\rfloor$ guards has the following steps. Construct a channel $C$ that joins the hole to the exterior boundary of $P$, in such a way that at most one additional vertex is created. Then remove $C$, producing a new polygon $P^{1}$ with $n+1$ vertices and no holes. This polygon can be guarded by $\left.(n+1) / 3\right\rfloor$ guards. The only part of the original polygon left unguarded is the channel $C$. To ensure that $C$ is also seen by at least one guard, we associate with $C$ during construction a triangle $T$ in the new polygon $P^{1}$. T has the property that a guard arbitrarily placed within it can see the entire channel in the original polygon. When $P^{1}$ is triangulated to place the guards, we force $T$ to be one of the triangles. After three-coloring, one of $T$ 's vertices is colored by the least-used color. The guard placed at this vertex sees all of $C$ and the $\lfloor(n+1) / 3\rfloor$ guards placed can then see not only $P^{1}$ but also all of the original polygon $P$.

We describe three methods of constructing $C$ such that:

(1) The net gain in vertices is at most one.

(2) A triangle in the remaining hole-free polygon sees all of $C$.

All the methods involve rotations of a ray around a source point. We adopt the convention that if the ray hits two or more vertices simultaneously, then the "first vertex encountered" refers to the vertex closest to the source point. In the following let the vertices on the exterior boundary $B$ be $p_{0}, \ldots, p_{m-1}$ in clockwise order and let the vertices on the hole $H$ be $h_{0}, \ldots, h_{n-m-1}$ in counterclockwise order. The first way to construct a channel is to pick an edge $h_{k-1} h_{k}$, where $h_{k}$ is a reflex vertex of $P$ on $H$, and extend it through $h_{k}$ until it hits the exterior boundary at a point $q$ on edge $p_{i} p_{i-1}$ (see Fig. 1). Create a new vertex $q^{\prime}$ a distance of $\varepsilon$ away from $q$ in the direction of $p_{-1}$ and connect $q^{\prime}$ to $h_{k+1} . C$ is the area $h_{k}, q, q^{\prime}, h_{k+1}$, The edge $q q^{\prime}$ has length $\varepsilon$ and is called the $\varepsilon$-side of $C$. The new polygon $P^{1}$ has vertices $p_{1}, \ldots, p_{i-1}, q^{\prime}, h_{k+1}, \ldots h_{k-1}, q, p_{i}, \ldots, p_{m}$, using modular arithmetic on the indices. The first property of a channel construction is satisfied because two new vertices $q$ and $q^{\prime}$ are present, but $h_{k}$ now lies interior to the edge $h_{k-1} q$ and is no longer a vertex. To satisfy the second property, a triangle $T$ is required. If $T$ can be found such that the area of $C \cup T$ is convex and empty, then every vertex of $T$ is guaranteed to see all of $C$ and the second property of a channel construction is satisfied. We choose as the base of $T$ one side of $C$. In this construction $q^{\prime} h_{k+1}$ is that side. A third vertex $v \notin q^{\prime} h_{k+1}$ to serve as the apex of $T$ must be found. Choose $v$ as the first vertex encountered when $h_{k+1} q$ rotates counterclockwise around $h_{k+1}$ with $q$ sliding along its edge toward $p_{i-1}$. If the quadrilateral $Q=\left(h_{k+1}, h_{k}, q, v\right)$ is convex and empty, then area $C \cup T$ is guaranteed to be 


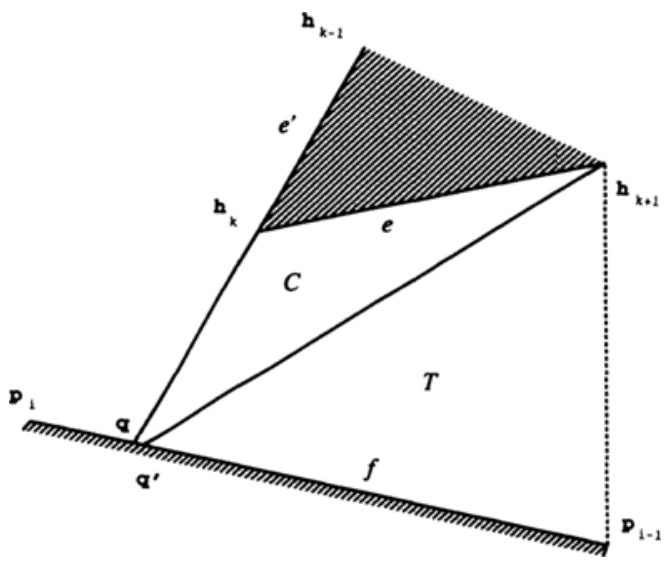

Fig. 1. Edge $h_{k-1} h_{k}$ is extended through $h_{k}$.

convex and empty. Above we extended edge $h_{k-1} h_{k}$ through $h_{k}$. A symmetric construction can be produced if we instead extend edge $h_{k+1} h_{k}$ through $h_{k}$.

In Case 2.1 we formalize the conditions that must hold for this type of construction to be possible. We let $h_{1}$ represent a vertex adjacent to $h_{k}$ in either direction. In the forward version of Case $2.1, h_{l}$ lies in a counterclockwise direction from $h_{k}$ and in the backward version of Case $2.1, h_{l}$ lies in a clockwise direction from $h_{k}$. Similarly vertex $p_{j}$ is adjacent to $p_{i}$. We consolidate the two versions into one case:

Case 2.1. A reflex vertex $h_{k}$ of $P$ on $H$ with incident edges $e=h_{k} h_{l}$ and $e^{\prime}$ and an edge $f=p_{i} p_{j}$ on $B$ exists such that the following conditions hold:

- The extension of $e^{\prime}$ through $h_{k}$ hits edge $f$ on $B$ at $q \neq p_{j}$.

- A half-plane defined by $\overline{h_{k} q}$ contains both $e$ and $p_{j}$.

- A vertex $v$ on $H$ or $B$ and a quadrilateral $Q=\left(h_{l}, h_{k}, q, v\right)$ exist such that:

1. $v \notin \overline{q h_{l}}$.

2. $v$ is the first vertex encountered by $h_{l} q$ as $q$ slides along $f$ toward $p_{j}$.

3. $Q$ is convex.

4. $Q$ has an empty interior.

Note that it is possible within Case 2.1 for the extension from $h_{k}$ to touch a vertex on $B$. In the special case where $q$ coincides with a vertex $p_{i}$ and $h_{k}, q$, and $p_{i-1}$ do not form a right turn, the construction results in a polygon $P^{1}$ with $n$ vertices. If the extension from $h_{k}$ first touches $B$ at a vertex $p_{j}$ and $h_{k}, p_{j}$, and $p_{j-1}$ form a right turn, then the extension is continued. The removal of $C$ divides $P$ into pieces. These pieces are triangulated separately and then joined at $p_{j}$ before the triangulation graph is three-colored.

The second method of constructing a channel is to connect vertex $h_{k}$ directly to $p_{i}$, create vertex $p_{i}^{\prime}$ a distance of $\varepsilon$ away from $p_{i}$ in the direction of $p_{i-1}$ and 

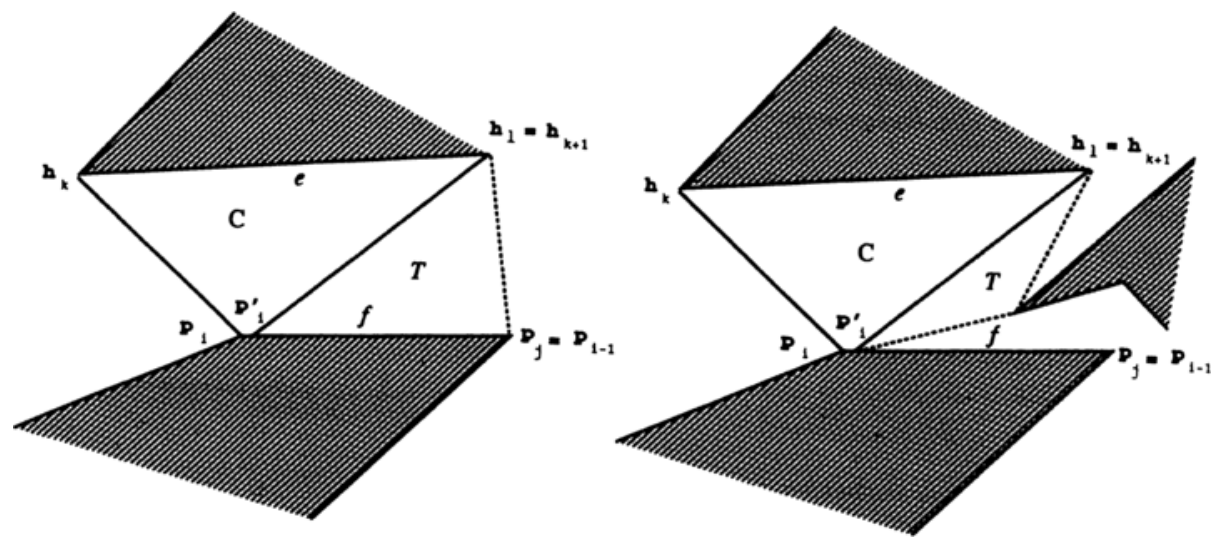

Fig. 2. Vertex $h_{k}$ is connected directly to vertex $p_{i}$.

connect it to $h_{k+1}$ (see Fig. 2). $C$ is the area $h_{k}, p_{i}, p_{i}^{\prime}, h_{k+1}$ with $\varepsilon$-side $p_{i} p_{i}^{\prime}$. The base of triangle $T$ lies along the side $p_{i}^{\prime} h_{k+1}$ and the third vertex $v$ is picked so that $Q=\left(h_{k+1}, h_{k}, p_{i}, v\right)$ is empty and convex. A backward version of the construction ensues if we instead place vertex $p_{i}^{\prime}$ in the direction of $p_{i+1}$ and connect it to $h_{k-1}$. This type of construction is applicable in the following case:

Case 2.2. An edge $e=h_{k} h_{l}$ on $H$ and an edge $f=p_{i} p_{j}$ on $B$ exist such that the following conditions hold:

- A half-plane defined by $\overline{h_{k} p_{i}}$ contains both $e$ and $p_{j}$.

- A vertex $v$ on $H$ or $B$ and a quadrilateral $Q=\left(h_{l}, h_{k}, p_{i}, v\right)$ exist such that:

1. $v \notin \overline{p_{i} h_{l}}$.

2. $v$ is the first vertex encountered by $h_{l} p_{i}$ as $p_{i}$ slides along $f$ toward $p_{j}$.

3. $Q$ is convex.

4. $Q$ has empty interior.

For the third and final method of constructing $C$, we extend an edge from a vertex $p_{i}$ on the exterior boundary until it hits an edge $e$ on $H$ at a point $k$ (see Fig. 3). Create a new vertex $p_{i}^{\prime}$ next to $p_{i}$ and connect it to the endpoint of $e$.

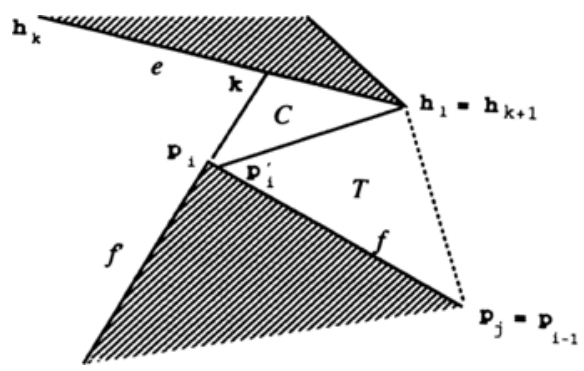

Fig. 3. Edge $p_{i-1} p_{i}$ is extended through $p_{i}$. 
$C$ has $\varepsilon$-side $p_{i} p_{i}^{\prime}$. A forward or backward version of the construction is produced depending on which incident edge is extended from $p_{i}$. This type of construction is applicable in the following case:

Case 2.3. An edge $e=h_{k} h_{l}$ on $H$ and a reflex vertex $p_{i}$ with incident edges $f$ and $f^{\prime}$ on $B$ exist such that the following conditions hold:

- The extension of $f^{\prime}$ through $p_{i}$ hits $e$ at $k \neq h_{l}$.

- A half-plane defined by $\overline{p_{i} k}$ contains both $f$ and $h_{l}$.

- A vertex $v$ on $H$ or $B$ and a quadrilateral $Q=\left(h_{l}, k, p_{i}, v\right)$ exist such that:

1. $v \notin \overline{p_{i} h_{l}}$.

2. $v$ is the first vertex encountered by $h_{l} p_{i}$ as $p_{i}$ slides along $f$ toward $p_{j}$.

3. $Q$ is convex.

4. $Q$ has an empty interior.

Each one of the above cases was specified to allow a corresponding type of channel to be constructed between an edge on $H$ and an edge on $B$. The $\varepsilon$-side of $C$ always lies on $B$.

Lemma 2.1. If one of the cases above holds for a polygon $P$ with one hole, then a channel can be removed from $P$ in such $a$ way that the new polygon $P^{1}$ has:

- $n+1$ vertices.

- No hole.

- A specific triangle $T$ from whose vertices the area of the removed channel is visible in $P$.

Proof. In each case the construction adds one vertex to the total, thus the number of vertices is $n+1$. Each constructed channel has one side along the hole and an $\varepsilon$-side along the exterior boundary. Thus no hole exists when the channel is removed. One side of $T$ is shared by $C$, guaranteeing that the two endpoints see all of $C$. The following method finds the third vertex $v$ of $T$. Rotate the edge shared by $C$ and $T$ around the endpoint situated on $H$. Let the other endpoint slide along the boundary edge on which it is situated. The first vertex encountered by the rotating edge is $v$. This vertex could be the endpoint of the boundary edge (see Fig. 1) or some other vertex (see Fig. 4). The condition that $Q$ must be convex guarantees that $v$ is encountered by the rotating edge before the rotation is carried past an extension of the edge shared by $C$ and $H$ (see Fig. 5). Thus the third vertex of $T$ sees all of $C$.

Lemma 2.1 guarantees that if one of the Cases 2.1-2.3 holds, then a channel with the required properties can be constructed. To prove that any hole can be connected to the exterior by a channel, it remains to show that in every polygon with one hole two edges (one on the hole and one on the exterior boundary) exist such that one of the cases is applicable. Before proceeding to this proof, it is helpful to understand what happens when none of the cases applies to two specified edges, in 


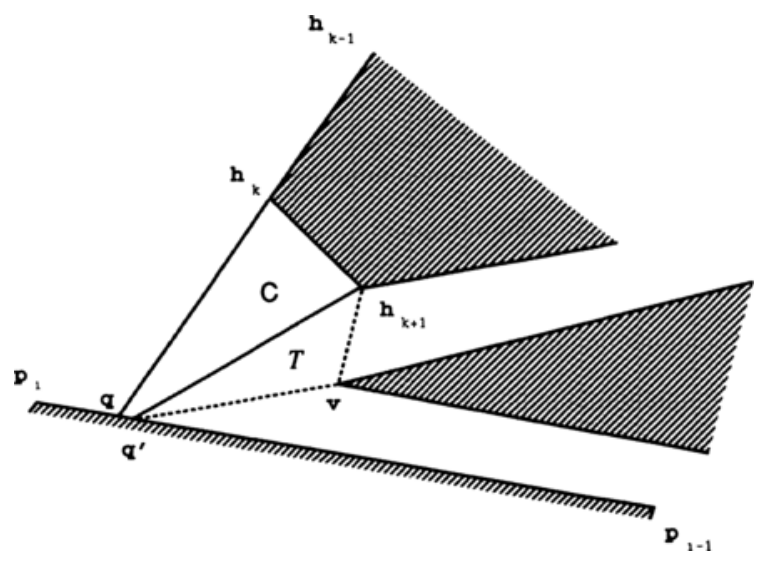

Fig. 4. The first vertex encountered as $h_{k+1} q^{\prime}$ rotates toward an extension of $h_{k} h_{k+1}$ is $v$.

particular when vertex $v$, as specified in the conditions for Cases $2.1-2.3$, does not exist.

We begin with the forward version of Case 2.1. Let $h$ and $p$ represent $h_{k}$ and $q$, respectively, and assume that the first two conditions of Case 2.1 hold with $e$ incident to $h$ in the counterclockwise direction. For Case 2.1 not to apply, it must be true that the requisite vertex $v$ does not exist. Let $l_{h}$ be a line through $e$ and let $l_{p}$ be a line through $f$ (see Fig. 6). If $l_{h}$ and $l_{p}$ are parallel or if they intersect at a point $z$ on the opposite side of $h p$ from $h_{l}$, then quadrilateral $Q^{\prime}=\left(h_{l}, h, p, p_{j}\right)$ is convex (see Fig. 6(a)). In this situation one or more vertices must lie inside $Q^{\prime}$, or $Q^{\prime}$ would be both empty and convex. At least one of these vertices must also lie in the triangle $\left(h, p, h_{l}\right)$, since otherwise one vertex $v$ could be picked from among the vertices in $Q^{\prime}$ in such a way that quadrilateral $\left(h_{l}, h, p, v\right)$ is empty and convex and $v \notin \overline{p h}$. If instead $z$ and $h_{l}$ lie on the same side of $h p$, then $Q^{\prime}$ may still be

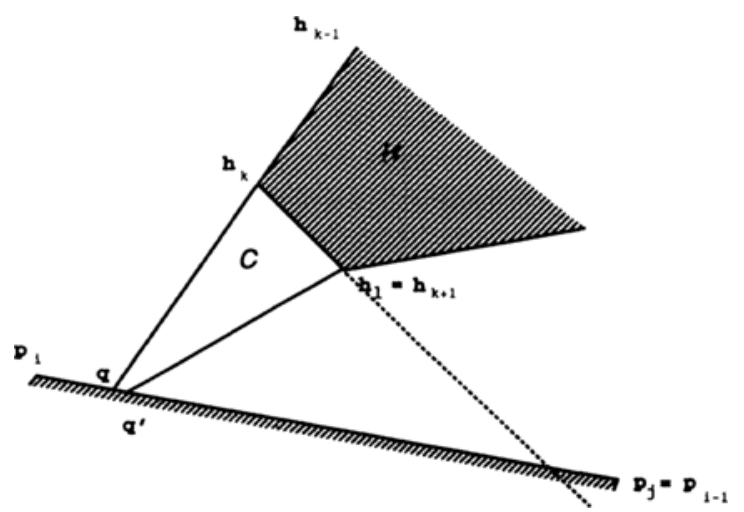

Fig. 5. If a vertex $v$ is not found before edge $h_{k+1} q^{\prime}$ has rotated past the extension of $h_{k} h_{k+1}$ through $h_{\mathrm{k}+1}$, then $Q$ is not convex. 


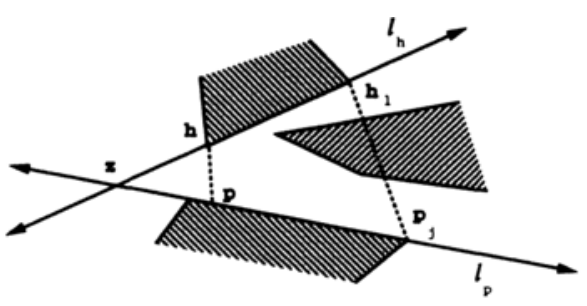

(a)

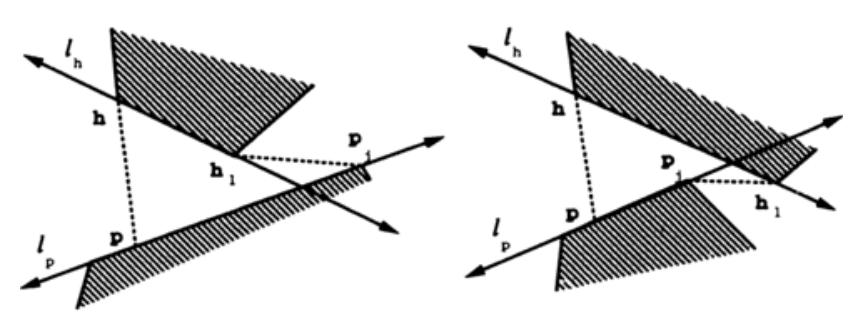

(b)

Fig. 6. (a) $Q^{\prime}$ is nonempty. (b) $Q^{\prime}$ is nonconvex.

nonempty but it is also possible that $Q^{\prime}$ is empty and nonconvex (see Fig. 6(b)). In $Q^{\prime}$ the interior angles at both $h$ and $p$ are convex since the first condition of Case 2.1 was assumed true. Either $h_{l}$ or $p_{j}$ must then be a reflex vertex.

We conclude from the preceding paragraph that for the forward version of Case 2.1 to fail either some vertex lies in triangle $\left(h, p, h_{l}\right)$ or $Q^{\prime}$ has a reflex angle at $h_{l}$ or $p_{j}$. The forward versions of Cases 2.2 and 2.3 fail in the same situations: in Case $2.2 h$ and $p$ represent two vertices $h_{k}$ and $p_{i}$, chosen such that the first condition of the case holds; in Case $2.3 h$ and $p$ represent $k$ and $p_{i}$ such that the first two conditions of the case hold.

We now assess the feasibility of the backward versions of Cases 2.1-2.3, where the forward versions are already known to fail. This is more complicated because we do not make any assumptions about the characteristics of $h_{l}$ and $p_{j}$ and the reasons for failure need not involve the existence of an empty convex quadrilateral $Q$. Therefore, we determine what the situation should be for one of the backward versions to apply. Specifically, if $Q^{\prime}$ as described above is empty and nonconvex with a reflex angle at $h_{l}$, then if an extension of the counterclockwise edge through $h_{l}$ hits the edge $p, p_{j}$, a vertex $v$ exists such that $Q=\left(h, h_{l}, q, v\right)$ is convex and empty and Case 2.1 applies (see Fig. $7(\mathrm{a})$ ). If $Q^{\prime}$ is empty and nonconvex with a reflex angle at $p_{j}$ (see Fig. 7(b)), then if an extension of the counterclockwise edge from $p_{j}$ hits a point $k$ on $h h_{l}$, a vertex $v$ exists such that $Q=\left(h, k, p_{j}, v\right)$ is convex and empty and Case 2.3 applies. Finally if $Q$ is convex and nonempty (see Fig. 7(c)) and an extension of the counterclockwise edge from $p_{j}$ hits $e$ at point $k$ on $h h_{l}$, then Case 2.3 applies if none of the vertices inside $Q$ lie in the triangle $\left(p_{j}, k, h\right)$. 


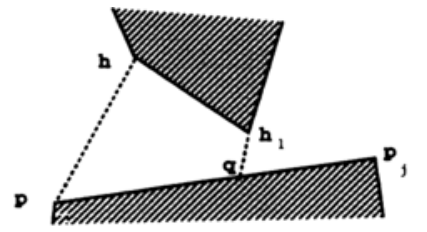

(a)

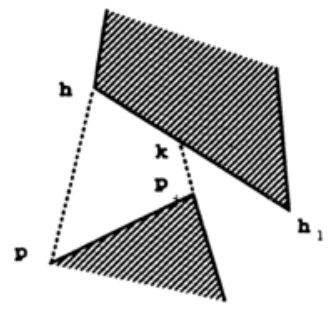

(b)

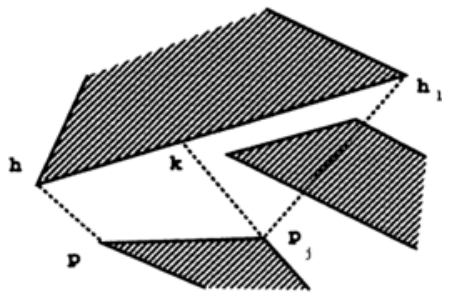

(c)

Fig. 7. (a) Case 2.1 applies. (b) and (c) Case 2.3 applies.

In conclusion, we stress that the obstruction of triangle $\left(h_{l}, h, p\right)$ or nonconvexity of $Q^{\prime}$ does not preclude the existence of an applicable case (in the situations above the backward versions can still be applied), but if no applicable case exists, then it must be true that either some vertex lies in triangle $\left(h_{l}, p, h\right)$ or $Q^{\prime}$ has a reflex vertex.

We are now prepared to prove the following lemma:

Lemma 2.2. At least one of the Cases 2.1-2.3 applies to any polygon with one hole.

Proof. The format of the proof is an algorithm which finds an edge $e$ on $H$ for which one of the cases applies. The strategy of the algorithm is to isolate two reflex vertices $a$ and $b$ of $P$ on $H$ with two corresponding points $r$ and $s$ on $B$ in such a way that one of the cases will apply in the area of $P$ which lies between the two segments $a r$ and $b s$ (see Fig. 8): $a$ must be chosen so that the extension of the edge clockwise from $a$ through $a$ intersects $B$ without intersecting any other part of $H ; b$ must be chosen so that the extension of the edge counterclockwise from $b$ through $b$ intersects $B$ without intersecting any other part of $H$ and at least one vertex lies on $B$ between $r$ and $s$. To this end, consider the relative convex hull of $H$ given $B$, defined as the minimum perimeter polygon containing $H$ constrained to lie in $B$. (For a description of relative convex hulls and their computation, see [9].) The extension of an edge from a vertex which lies both on $H$ and the relative convex hull of $H$ given $B$ cannot intersect $H$. Let $a$ be the leftmost such vertex and let $r$ be the first intersection point between $B$ and the extension from $a$, such that $a, r$, and $p_{i-1}$ form a left turn, where $p_{i} p_{i-1}$ is the edge on which $r$ 


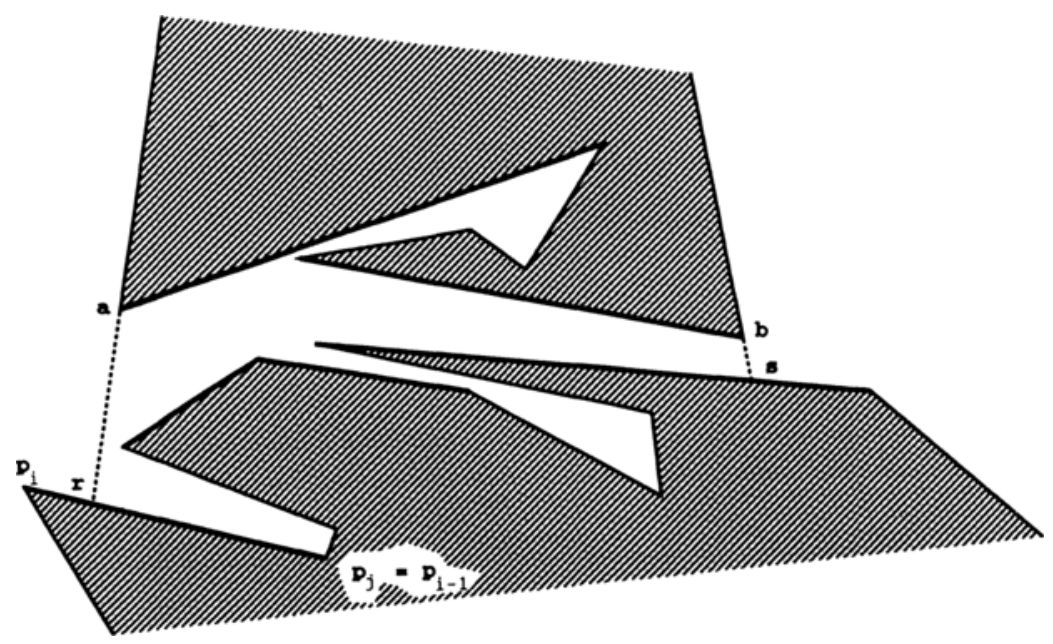

Fig. 8. The area between $a r$ and $b s$ is searched for an applicable case.

lies. To locate $b$, traverse the vertices of the relative convex hull of $H$ given $B$ in a counterclockwise direction from $a$ and pick as $b$ the first vertex on $H$ such that an extension of the edge counterclockwise of $b$ through $b$ intersects $B$ at a point $s$ without intersecting the segment $a p_{i-1}$. Vertex $b$ must exist. We walk around the relative convex hull of $H$ given $B$ and stop to check every vertex which lies on $H$. An extension of the counterclockwise edge from such a vertex will not hit $H$. However, we must also make sure that it does not intersect $a p_{i-1}$. Let the direction of $\overrightarrow{a r}$ be $d$ and let the direction of $\overrightarrow{r p_{i-1}}$ be $d^{\prime}$. Since $a, r$, and $p_{i-1}$ form a left turn, it follows that $d^{\prime}<d+180$. If we start the traversal at $a$ facing in direction $d$ and if we complete the traversal and return to $a$, then we will have turned $360^{\circ}$. Consequently we must at some vertex $w$ be facing in the direction $d^{\prime \prime}$ where $d^{\prime \prime}>d^{\prime}+180$ or we could never return to $a$. An extension of the edge counterclockwise from $w$ through $w$ cannot intersect $a p_{i-1}$. Segment $b s$ lies in a counterclockwise direction from $a r$.

Having delimited the search area, we start the search by placing a segment $h^{\prime} p^{\prime}$ so that $h^{\prime}$ coincides with $a$ and $p^{\prime}$ coincides with $r$. The segment $h^{\prime} p^{\prime}$ will be walked along the two chains in steps. This move is always in a counterclockwise direction with $h^{\prime} p^{\prime}$ growing and shrinking to keep one endpoint on each chain. At each step, check to see whether any case applies. If no case applies, then either $h^{\prime}$ or $p^{\prime}$ moves to a new vertex. The move guarantees that $h^{\prime}$ and $p^{\prime}$ see each other all times. Specifically, let $e$ be the edge incident to $h^{\prime}$ in a counterclockwise direction and let $f$ represent $p^{\prime} p_{i-1}$. First try to apply Cases 2.1 and 2.3 using edges $e$ and $f$. If unsuccessful, then one of the following situations holds:

(1) $Q$ is nonempty.

(2) $Q$ is empty with a refiex angle at $h_{l}$.

(3) $Q$ is empty with a reflex vertex at $p_{j}$. 


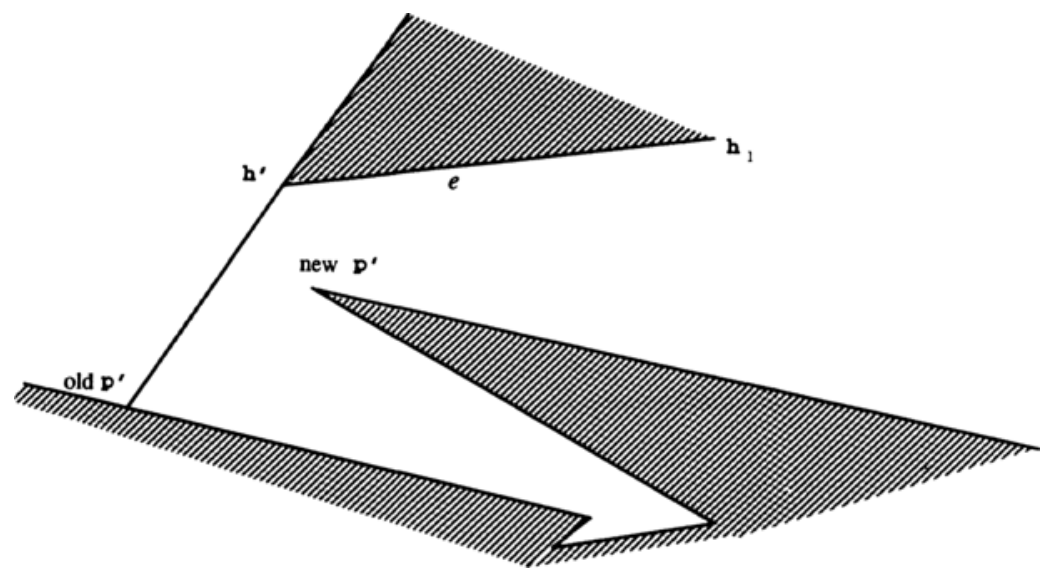

Fig. 9. The first encountered vertex as $h^{\prime} p^{\prime}$ rotates lies on the exterior boundary. This vertex becomes the new $p^{\prime}$.

In either case we wish to move one endpoint of $h^{\prime} p^{\prime}$ and try again. To decide what the particular move should be, we first rotate $h^{\prime} p^{\prime}$ around $p^{\prime}$, letting $h^{\prime}$ slide along $e$ in a counterclockwise direction. Let $x$ be the first vertex encountered by the rotating $h^{\prime} p^{\prime}$. Vertex $x$ will either be found before the endpoint of $e$ has been reached or $x$ will be this endpoint. If $x$ lies on $B$, we take a step by moving $p^{\prime}$ to $x$, leaving $h^{\prime}$ in its old position (see Fig. 9). If $x$ lies on $H$, then $h^{\prime}$ is moved to $x$ and $p^{\prime}$ remains unchanged (see Figs. 10 and 11).

Note that the choice of $x$ ensures that $x$ sees both $h^{\prime}$ and $p^{\prime}$ before the move and consequently after the move $h^{\prime}$ and $p^{\prime}$ see each other. The choice of $x$ also implies that if $x$ lies on $B$, then $x$ is a reflex vertex.

After the move, we try again to apply the cases in the following order:

- Try the forward version of Case 2.2 if $p^{\prime} \neq r$ and construct a channel if possible.

- Try the forward version of Case 2.1 if $p^{\prime}=r$, using $q=p^{\prime}$, but do not construct a channel. While $p^{\prime}=r$, it is possible that Case 2.2 does not apply because

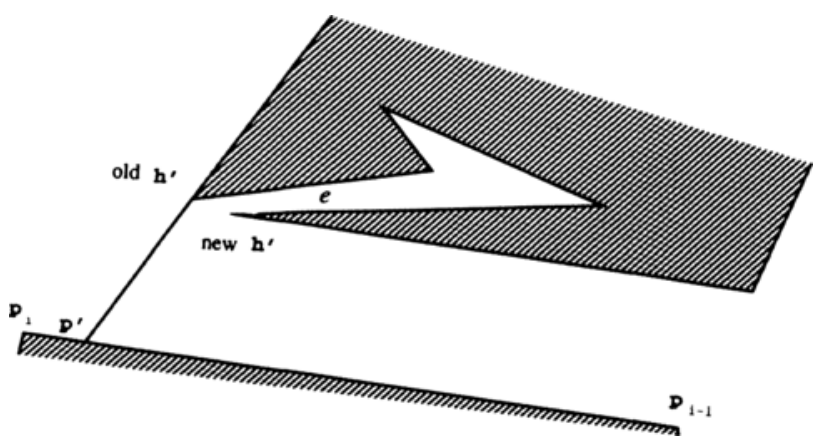

Fig. 10. The first encountered vertex is a hole vertex. It becomes the new $h^{\prime}$. 


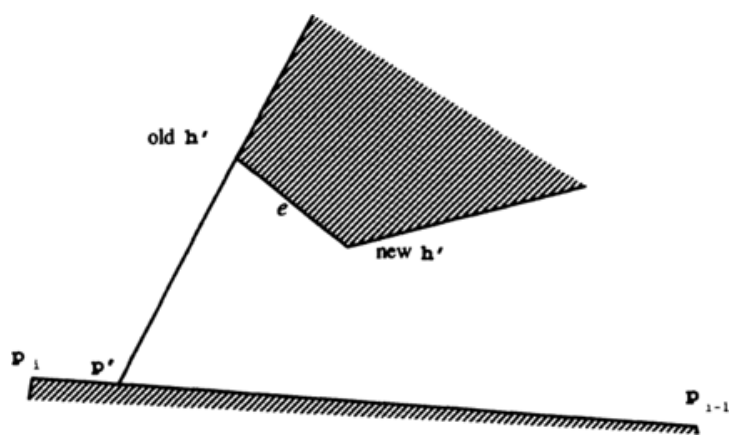

Fig. 11. The endpoint of $e$ becomes the new $h^{\prime}$.

$h^{\prime} p_{i}$ may be obstructed (see Fig. 12) and no nonempty quadrilateral $Q$ exists. Case 2.2 is used to find a vertex $x$ to which $h^{\prime}$ or $p^{\prime}$ will be moved in the next step. If no vertex $v$ exists such that quadrilateral $Q=\left(h_{l}, h^{\prime}, p^{\prime}, v\right)$ is convex and empty, then $x$ is found by the rotation of $h^{\prime} p^{\prime}$ as above. If vertex $v$ exists and $v \neq p_{i-1}$, then $v$ is the vertex to which $h^{\prime}$ or $p^{\prime}$ will be moved in the next step. Finally, if $v=p_{i-1}$, then the backward version of Case 2.2 will apply when tried below and no further move is needed for $h^{\prime} p^{\prime}$.

- Try the backward version of Case 2.1 if $x=h_{l}$ and the extension of $e^{\prime}$ hits $f$ and construct a channel if possible.

- Try the backward version of Case 2.2 if $v=p_{i-1}$ and construct a channel if possible.

- Try the forward version of Case 2.3 if $p^{\prime}$ is a vertex and construct a channel if possible.

- Try the backward version of Case 2.3 with an extension of the edge counterclockwise from $p^{\prime}$ if $p^{\prime}$ is a vertex and construct a channel if possible.

- Try the backward version of Case 2.3 and construct a channel if possible.

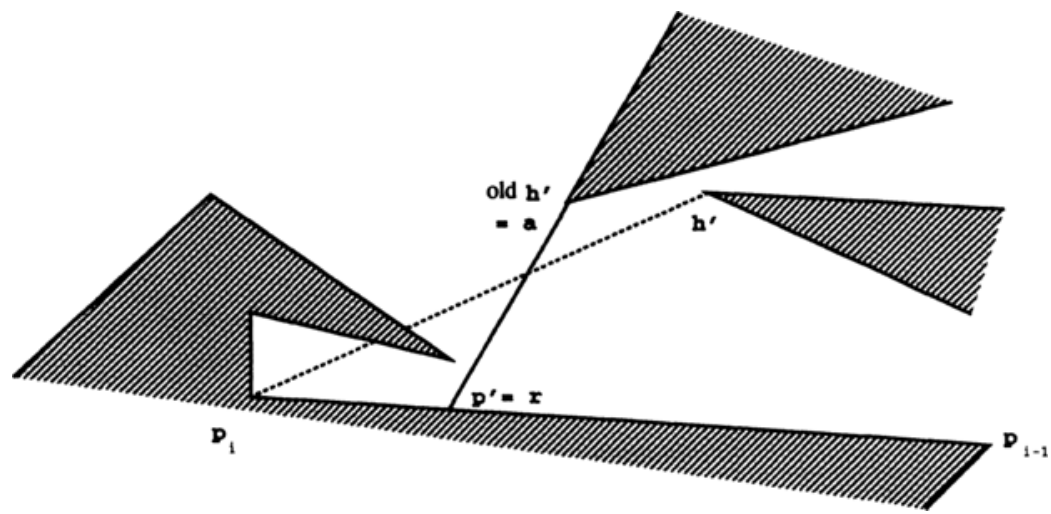

Fig. 12. While $p^{\prime}=r$ it is possible that $h^{\prime}$ does not see $p_{i}$. 
If none of the above steps was successful, i.e., none of the Cases 2.1-2.3 were applicable, then in particular the forward versions of the three cases all failed. It follows, as discussed in the preamble to Lemma 2.2, that either some vertex $x$ is present in the triangle $\left(h^{\prime}, p^{\prime}, h_{l}\right)$ or the quadrilateral $\left(h_{l}, h^{\prime}, p^{\prime}, p_{i-1}\right)$ is nonconvex. We again use rotations of $h^{\prime} p^{\prime}$ to find $x$ and move either $h^{\prime}$ or $p^{\prime}$ one step in a counterclockwise direction. If triangle $\left(h_{l}, h^{\prime}, p^{\prime}\right)$ is obstructed, then $x$ is the first obstructing vertex. If triangle $\left(h_{l}, h^{\prime}, p^{\prime}\right)$ is empty and quadrilateral $\left(h_{l}, h^{\prime}, p^{\prime}, p_{i-1}\right)$ is nonconvex, then $h_{l}$ is the new $h^{\prime}$. Each time we fail to find an applicable case, we move one step closer to the segment $b s$. We make the following three observations:

1. Either an applicable case exists or a move of $p^{\prime}$ or $h^{\prime}$ is made.

2. Since vertex $b$ is chosen in such a way that no part of the polygon intersects $b s$, a vertex in a counterclockwise direction from $b$ or $s$ cannot be chosen as $x$ until $h^{\prime}$ is positioned at $b$.

3. In any step where triangle $\left(h_{l}, h^{\prime}, p^{\prime}\right)$ is obstructed, vertex $b$ cannot be the first obstructing vertex encountered as $h^{\prime} p^{\prime}$ is rotated. Since $b s$ is an extension of the edge counterclockwise from $b$, the other endpoint of the edge clockwise from $b$ would be encountered first. Thus the only way that $b$ can be chosen as the new $h^{\prime}$ is when $b$ is the current $h_{l}$ and quadrilateral $\left(h_{l}, h^{\prime}, p^{\prime}, p_{i-1}\right)$ is nonconvex.

From the above observations it follows that if no applicable case is found, then

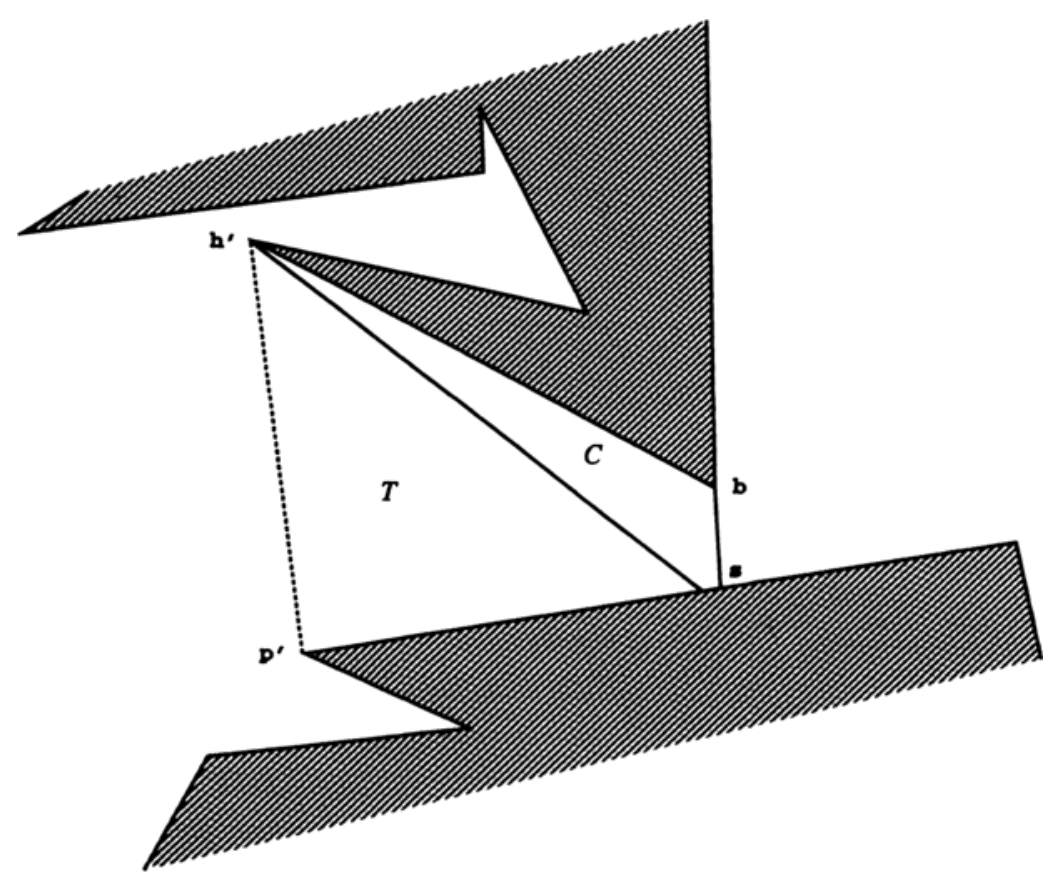

Fig. 13. Case 2.1 applies. 
segment $h^{\prime} p^{\prime}$ is moved in a counterclockwise direction until $h^{\prime}$ is located at the vertex adjacent to $b$ and $p^{\prime}$ is the endpoint of the edge on which $s$ lies (see Fig. 13). At this point the quadrilateral $\left(h^{\prime}, p^{\prime}, s, b\right)$ is convex and empty, since $b$ and $s$ are chosen so that no part of $P$ intersects $b s$. Our choice of $b$ and $s$ guarantees that Case 2.1 applies from $b$ with $v=p^{\prime}$ and the algorithm terminates successfully.

The algorithm is illustrated in Fig. 14, where we show each step taken by segment $h^{\prime} p^{\prime}$ in the search area from Fig. 8.

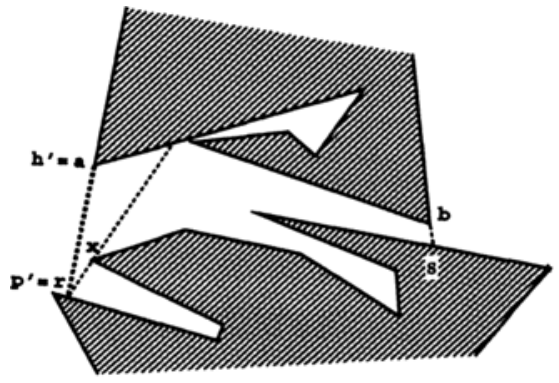

(a)

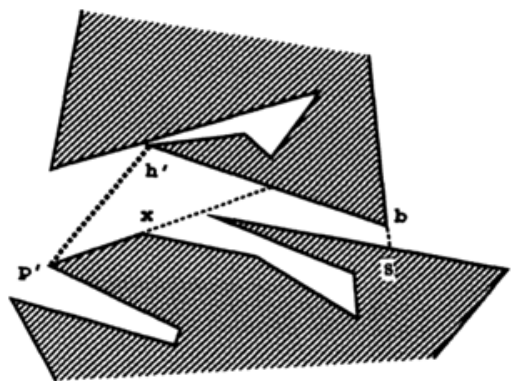

(c)

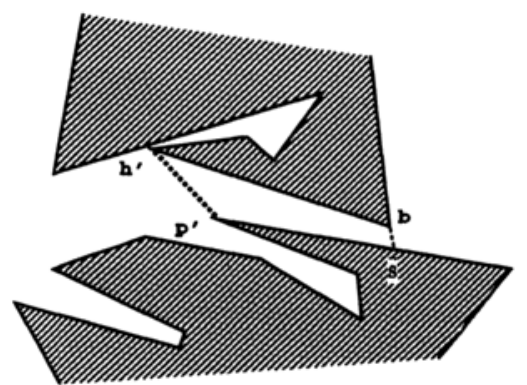

(e)

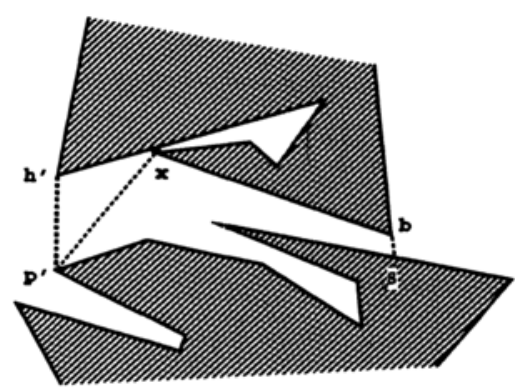

(b)

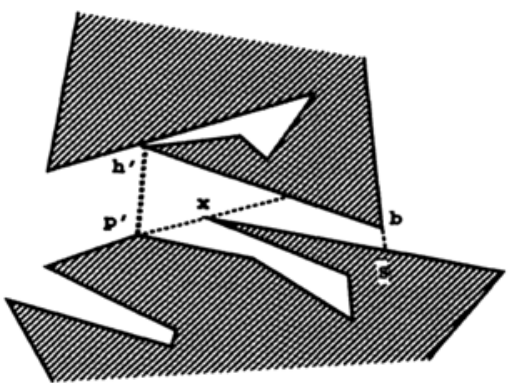

(d)

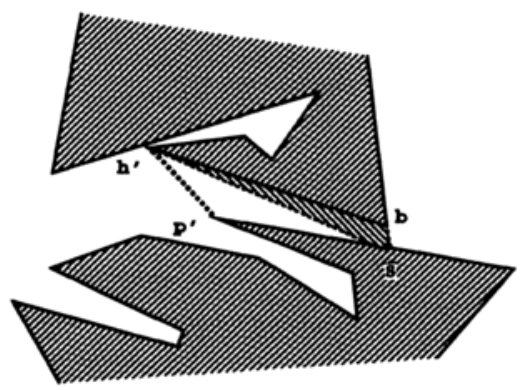

(f)

Fig. 14. The steps taken by $h^{\prime} p^{\prime}$ before an applicable case is found. The heavy dashed line indicates the current position of $h^{\prime} p^{\prime}$. In (a)-(d) the thin dashed line shows how far $h^{\prime} p^{\prime}$ must rotate before a vertex $x$ is encountered. In (e) the quadrilateral $\left(h^{\prime}, b, s, p^{\prime}\right)$ is convex and empty, Case 2.1 applies and the resulting channel is seen in (f). 


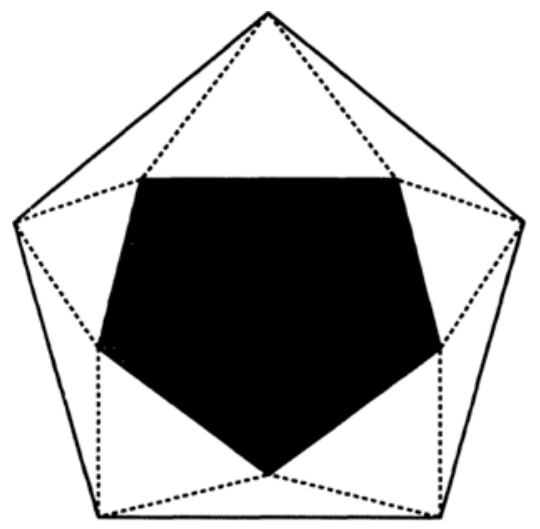

(a)

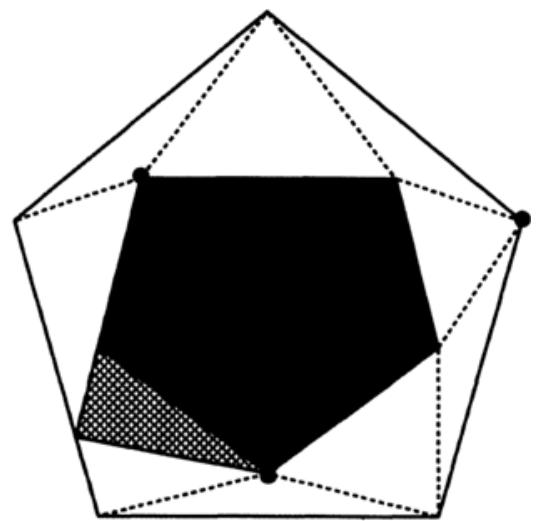

(b)

Fig. 15. (a) Triangulation where four combinatorial guards are necessary. (b) After the indicated channel is removed, guards are placed at the black vertices.

Lemmas 2.1 and 2.2 together prove the following theorem:

Theorem 2.3. In any polygon $P$ with $n$ vertices and one hole a channel can be removed in such $a$ way that the remaining polygon has:

- $n+1$ vertices.

- No hole.

- A triangle from whose vertices the area of the removed channel is visible in $P$.

The $\lfloor(n+1) / 3\rfloor$ bound on the number of guards required in a polygon with one hole follows from the theorem. While all the guards are vertex guards in $P^{s}$, not all these vertices exist in $P$. The guards in $P$ are therefore point guards.

We conclude this section by showing how our algorithm handles a polygon, whose triangulation is tough. As discussed in Section 1, a tough triangulation is one where one triangle must be left without a guard at one of its vertices in order not to use more than $\lfloor(n+1) / 3\rfloor$ guards. Figure $15(\mathrm{a})$ shows such a polygon with ten vertices. If we require every triangle to have a guard at one of its corners, i.e., if we require the guards to be combinatorial, then four guards are needed. Figure 15(b) shows the channel constructed for this polygon by our algorithm and also the resulting guard assignment. The removal of a channel from the polygon has the result that the triangulation of the new polygon contains one less triangle. The assigned guards are combinatorial in the hole-free polygon, but not in the original polygon. The problem of making sure that one triangle is guarded without having a guard at one of its vertices is handled automatically by the algorithm and does not present any problems.

\section{Guarding a Polygon with an Arbitrary Number of Holes}

When the polygon has $h$ holes, the construction of a channel as in Section 2 above, will be repeated $h$ times. Each construction and removal of a channel results in a 
new polygon with one additional vertex and one less hole. After $h$ repetitions we have a polygon with $n+h$ vertices and no holes. Inside this polygon a triangle for each channel will exist such that the vertices of the triangle see all of the area covered by the corresponding channel in the original polygon. In the remainder of this section we show exactly how the triangles and constructions are modified to ensure that these triangles exist. With the triangles in place, a guard assignment based on three-coloring in the hole-free polygon will cover the original polygon with holes. Since the new polygon has $n+h$ vertices, the number of guards is $\lfloor(n+h) / 3\rfloor$. These guards are vertex guards in the hole-free polygon, but point guards in the original polygon, since new vertices were added during the channel constructions.

To construct the first channel, we start the search at vertex $a$ of one hole and point $r$, which satisfy the same conditions as in the case of $h=1$. We place the segment $h^{\prime} p^{\prime}$ to coincide with ar and try to construct a channel. If no case applies, either $h^{\prime}$ or $p^{\prime}$ is moved in a counterclockwise direction as described in Section 2, but now $h^{\prime}$ may move to a different hole. This substitution does not affect the existence of an applicable construction; as long as no construction is found we keep moving $h^{\prime}$ or $p^{\prime}$ and eventually must reach a situation where the extension of the edge counterclockwise from a vertex $b$ through $b$ hits the boundary at $s$ and the quadrilateral $\left(h^{\prime}, b, s, p^{\prime}\right)$ is convex with an empty interior. At this point Case 2.1 applies. The vertex $b$ is not predetermined; instead we just test each new vertex adjacent to $h^{\prime}$ in a counterclockwise direction as a potential $b$. The reason for this change in procedure is that, with several holes, the exterior boundary may partially hide some holes from each other and as we move in a counterclockwise direction from $a$, even some holes on the relative convex hull may be skipped (see Fig. 16). At the point where a construction is found, one hole (not necessarily the hole where the search started) is joined to the boundary by a channel adding one vertex to the total. We then proceed to search for the next channel, starting at the point where we last switched from one hole to another, or, if no such switch occurred, we restart the search at a newly picked vertex $a$. Let the channels be $C_{1}, C_{2}, \ldots, C_{h}$. The construction and removal of the first channel $C_{1}$ results in a polygon $P^{1}$ with $n+1$ vertices and $h-1$ holes. Constructing and removing channel $C_{2}$ results in polygon $P^{2}$ with $n+2$ vertices and $h-2$ holes, etc., until we have a polygon $P^{h}$ with $n+h$ vertices and no holes. We must at this point also have $h$ triangles $T_{1}, T_{2}, \ldots, T_{h}$ inside $P^{h}$ such that guards anywhere inside these triangles can see all of the area covered by channels $C_{1}, \ldots, C_{h}$ in the original polygon $P$.

Our main concern with multiple holes is that once we have a completed channel and its associated triangle, the construction of a subsequent channel may interfere with the existing triangle. Let $t_{1}, t_{2}$, and $t_{3}$ be the vertices of triangle $T_{r}$ associated with channel $C_{r}$. The side $t_{1} t_{2}$ is the side shared with $C_{r}$ and $t_{1}$ was originally a hole vertex. Assume a channel $C_{s}(s>r)$ is constructed. After the construction of $C_{s}$ the following two conditions must hold:

1. Each vertex $t_{1}, t_{2}$, and $t_{3}$, if superimposed on the original polygon $P$, sees the area covered by $C_{r}$.

2. $T_{r}$ must exist within $P^{s}$ (and ultimately within $P^{h}$ ). 


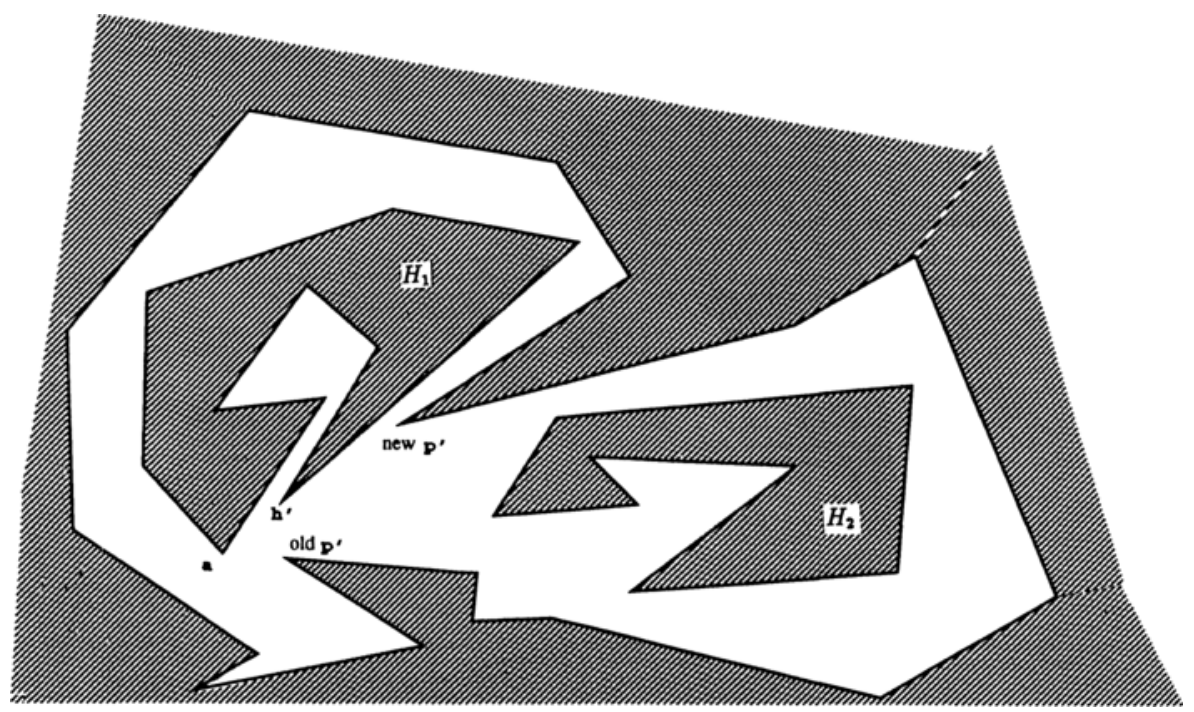

Fig. 16. No vertex on hole $H_{2}$ becomes $h^{\prime}$ since part of $B$ inserts itself between $H_{1}$ and $H_{2}$.

A triangle $T_{\mathrm{r}}$ may go through multiple changes during the course of the algorithm. Here we detail the types of changes that can occur as well as additional restrictions on some of the cases to guarantee that conditions 1 and 2 still hold at the end of the channel constructions. A triangle $T_{r}$ has one of two types. Either $t_{2} t_{3}$ is an edge on the exterior boundary forming an angle of less than $180^{\circ}$ with $t_{1} t_{2}$ (see Fig. 17), or $t_{2} t_{3}$ is interior to $P r$, in which case another boundary edge $t_{2} p_{j}$ also forming an angle of less than $180^{\circ}$ with $t_{1} t_{2}$ originates at $t_{2}$ (see Fig. 18). For condition 1 to be violated, one of the vertices of $T_{r}$ must have moved. Vertices are moved only in constructions where Cases 2.1 and 2.3 are applied. The construction used in Case 2.1 extends an edge from a reflex vertex on $H$ and the construction in Case 2.3 extends an edge from a reflex vertex on $B$. Vertex $t_{3}$ is the only vertex of $T_{r}$ that can lie on a hole. Thus if Case 2.1 was applied to construct $C_{s}$, then vertex $t_{3}$ on $H$ was moved. If Case 2.3 was applied, then vertex $t_{1}$ or $t_{3}$ (if $t_{3}$ lies on $B$ ) was moved. Vertex $t_{2}$ is never moved since $t_{2}$ is not a reflex vertex. For condition 2 to be violated, either a vertex has moved as described above or $C_{s}$ removed part of $T_{r}$ or both. $C_{s}$ has removed part of $T_{r}$ if the $\varepsilon$-side of $C_{s}$ is placed along edge $t_{1} t_{2}$ or $t_{2} t_{3}$ (or $t_{2} p_{j}$ in the second version of $T_{r}$ ). In the first version of $T_{r}$ an $\varepsilon$-side along edge $t_{1} t_{2}$ will cut off vertex $t_{1}$ from the rest of $T_{r}$ and an $\varepsilon$-side along $t_{2} t_{3}$ will cut off vertex $t_{3}$. In the second version of $T_{r}$ the vertices cut off are $t_{1}$ or $t_{2}$ in the first case and $t_{3}$ in the second case.

There are thus six instances in which the construction of $C_{s}$ interferes with the conditions that must hold for $T_{r}$ :

- Vertex $t_{3}$ lies on $B$ and is moved.

- Vertex $t_{3}$ lies on $H$ and is moved.

- Vertex $t_{1}$ is moved. 


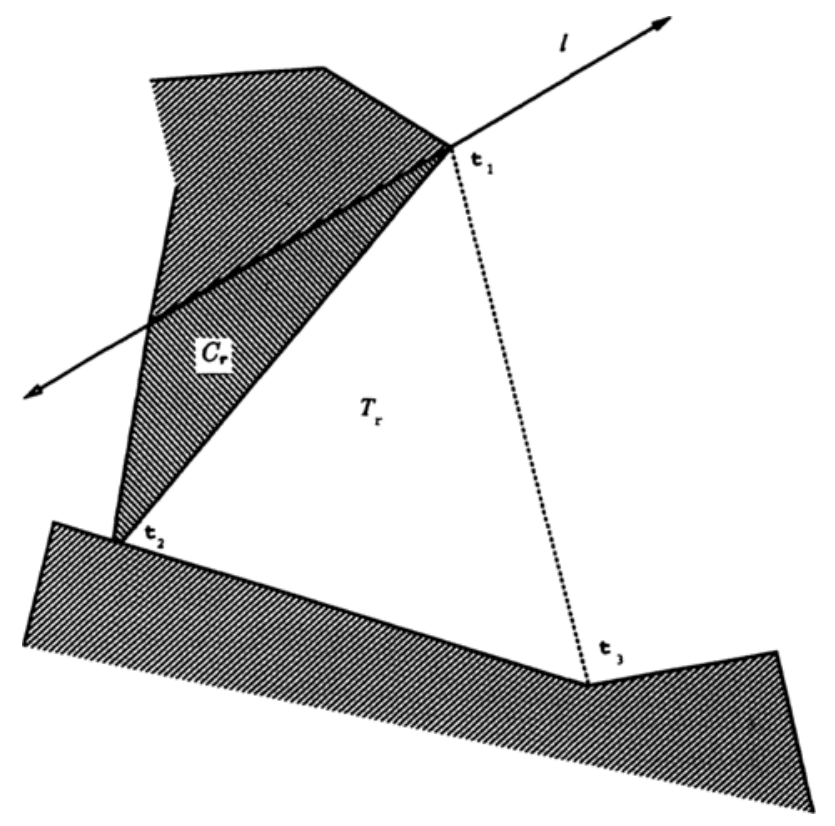

Fig. 17. The side $t_{2} t_{3}$ lies on the exterior boundary.

- Vertex $t_{3}$ is cut off.

- Vertex $t_{2}$ is cut off.

- Vertex $t_{1}$ is cut off.

We treat each instance as a separate case. Let $Q_{r}=\left(g_{r}, h_{r}, p_{r}, t_{3}\right)$ be the quadrilateral that was specified as convex and empty when $C_{r}$ was constructed. Similarly let $Q_{s}=\left(g_{s}, h_{s}, p_{s}, v_{s}\right)$ be the corresponding quadrilateral for $C_{s}$. Let $l_{r}$ and $l_{s}$ be lines through $g_{r} h_{r}$ and $g_{s} h_{s}$, respectively.

Instance 1. The triangle side $t_{2} t_{3}$ is an edge on the boundary. Vertex $t_{3}$ is moved.

Case 2.3 is applied to construct $C_{s}$ with the result that $Q_{r}$ becomes nonconvex or nonempty. When $t_{2} t_{3}$ is extended, $t_{3}$ moves away from its original position and the side $g_{r} t_{3}$ rotates around $g_{r}$ (see Fig. 19). If $Q_{r}$ becomes nonempty while still being convex, then some vertex $v$ in $Q_{r}$ exists such that $Q_{r}^{\prime}=\left(g_{r}, h_{r}, p_{r}, v\right)$ is empty and convex. Vertex $v$ replaces $t_{3}$, and a modified version of $T_{r}$ exists. If $Q_{r}$ should remain empty but become nonconvex, then the case that was applied to construct $C_{r}$ no longer applies. $Q_{r}$ becomes nonconvex when $t_{3}$ reaches point $z$, which is the intersection point between $l_{r}$ and a line $l_{p}$ through $t_{2} t_{3}$. Consequently, $Q_{r}$ may become nonconvex if $z$ and $t_{3}$ lie on the same side of $h_{r} p_{r}$. To prevent this problem, we further restrict the cases described in Section 2. In particular, we add the condition that if $t_{3}$ and $z$ lie on the same side of $h_{r} p_{r}$ and $t_{3}$ is chosen as the endpoint of the edge on which $p_{r}$ lies, then the triangle $\left(g_{r} t_{3} z\right)$ must not be 


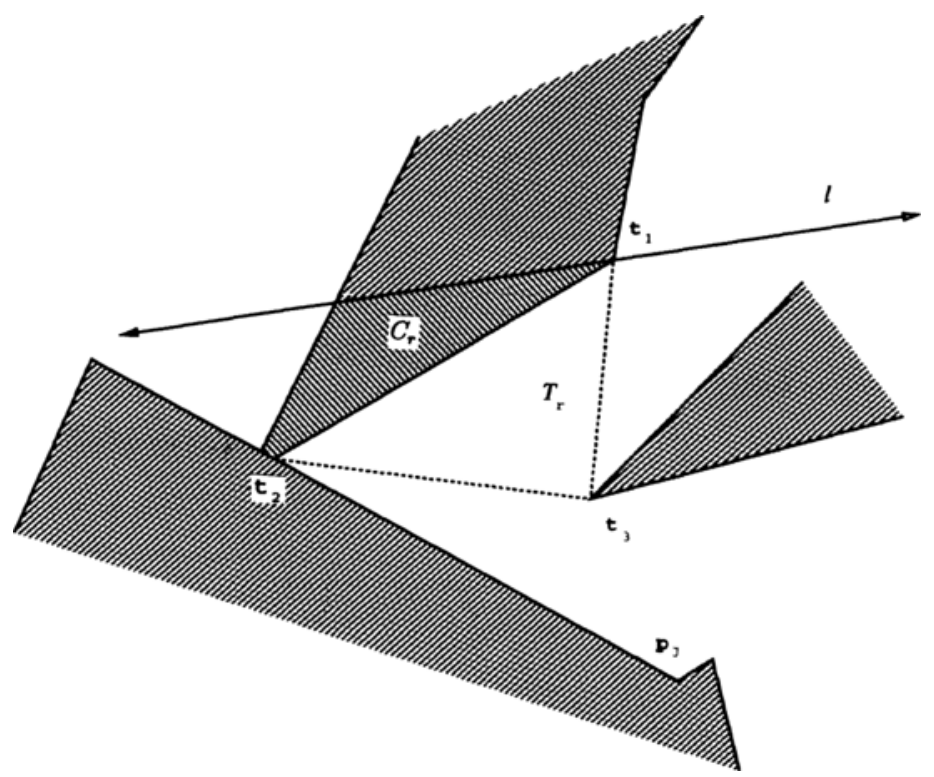

Fig. 18. The side $t_{2} t_{3}$ is interior to the polygon.

empty. An applicable case still always exists since when the quadrilateral $\left(g_{r}, h_{r}, p_{r}, t_{3}\right)$ is empty and convex and $z$ lies on same side of $h_{r} p_{r}$ as $t_{3}$, then a vertex $v$ exists such that $Q_{r}^{\prime}=\left(h_{r}, g_{r}, t_{3}, v\right)$ is empty and convex and Case 2.2 applies with $g_{r}$ as $h_{k}$ and $t_{3}$ as $p_{i}$.

Instance 2. The triangle side $t_{2} t_{3}$ is interior to the polygon. Vertex $t_{3}$ is moved.

Case 2.1 is applied to construct $C_{s}$ with the result that $Q_{r}$ changes shape. Assume first that the extension from $t_{3}$ hits edge $t_{2} p_{j}$. This edge will now

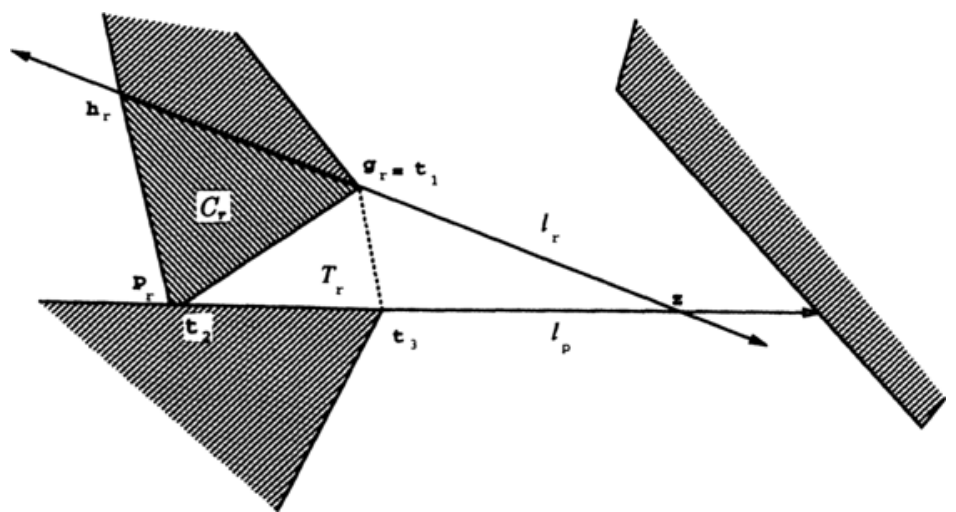

Fig. 19. Extending edge $t_{2} t_{3}$ so that $t_{3}$ ends up on the opposite side of $l$ makes $Q$, nonconvex. 


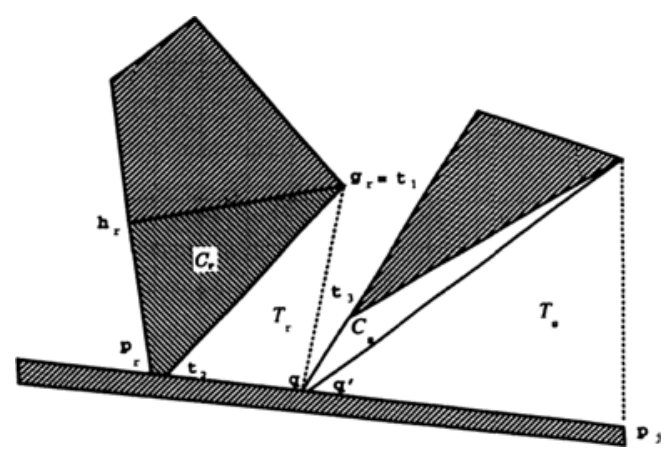

Fig. 20. The side clockwise from $t_{3}$ is extended through $t_{3}$, hitting edge $t_{2} p_{j}$.

have two new vertices: the relocated $t_{3}$ (called $q$ in Case 2.1) and $q^{\prime}$ which lies at a distance of $\varepsilon$ away from $q$. If $t_{2}$ and $q^{\prime}$ are on opposite sides of $q$ (see Fig. 20), then $Q_{r}=\left(g_{r}, h_{r}, p_{r}, t_{3}\right)$ remains empty and convex with $t_{3}$ in its new location, i.e., $t_{3}=q$. If $t_{2}$ and $q^{\prime}$ are on the same side of $q$ (see Fig. 21), then $Q_{r}^{\prime}=\left(g_{r}, h_{r}, p_{r}, q^{\prime}\right)$ is empty and convex. Vertex $q^{\prime}$ replaces $t_{3}$ and $T_{r}$ exists in $P_{s}$ in a modified form. The quadrilateral $\left(g_{s}, h_{s}, p_{s}, t_{1}\right)$ is convex, since $t_{3}$ is the first vertex encountered as $g_{r} p_{r}$ rotates around $g_{r}$. If it is not empty, then some vertex $v_{s}$ inside it can be picked such that $Q_{s}=\left(g_{s}, h_{s}, q, v_{s}\right)$ is empty and convex.

Assume instead that one or both extensions through $t_{3}$ hit edge $t_{1} t_{2}$ (see Fig. 22) and we apply the construction associated with Case 2.1. The result is that $T_{r}$ no longer exists in $P^{s}$. There are two possibilities. Either we just started the search for an applicable case with $t_{3}$ chosen as $a$ and point $r$ situated on $t_{1} t_{2}$, or the search started at a vertex in a clockwise direction from $t_{3}$. If our search just started, then our moving line segment $h^{\prime} p^{\prime}$ currently has $h^{\prime}=a$ and $p^{\prime}=r$. We take a step by moving $p^{\prime}$ to $t_{2}$ and continue the search. The move is in a counterclockwise direction and the new $p^{\prime}$ sees both $h^{\prime}$ and $r$. Since $t_{3}$ cannot play the role of both $a$ and $b$, an applicable case is still guaranteed to exist.

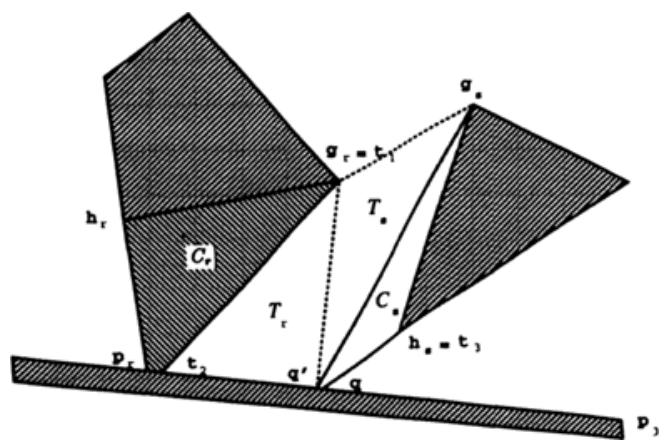

Fig. 21. The side counterclockwise from $t_{3}$ is extended through $t_{3}$, hitting edge $t_{2} p_{j}$. 


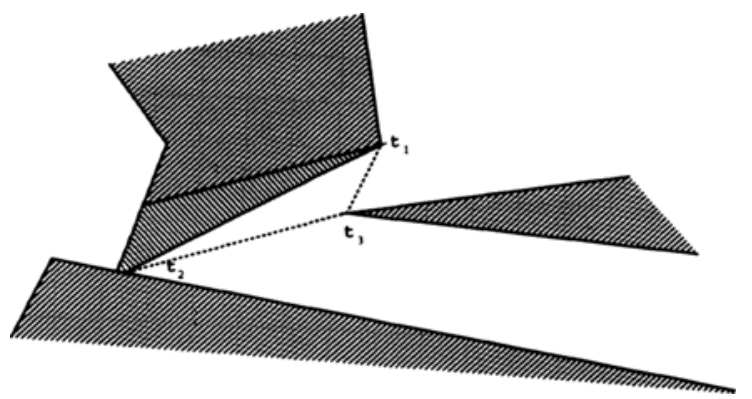

Fig. 22. An edge is extended through $t_{3}$, hitting side $t_{1} t_{2}$.

If instead our search started at some vertex in a clockwise direction from $t_{3}$, then segment $h^{\prime} p^{\prime}$ is currently positioned with $h^{\prime}$ at vertex $g_{s}$ and $p^{\prime}$ at vertex $t_{1}$. Let $l_{p}$ be a line through the edge incident to $t_{1}$ in a clockwise direction. If $l_{p}$ intersects $t_{2} t_{3}$ or if $t_{1}$ is convex, then an empty convex quadrilateral $\left(g_{s}, t_{3}, t_{1}, v\right)$ exists and the construction associated with Case 2.2, with edge $g_{s} t_{3}$ as $e$, applied in a previous step (see Fig. 23). Thus we must have moved forward in our search only if $l_{p}$ does not intersect $t_{2} t_{3}$ and $t_{1}$ is reflex. In this case no easy modification of $T_{r}$ exists, and $t_{3}$ may be designated $b$ so that we cannot move forward. We prevent this problem by disallowing the construction of $C_{r}$ and $T_{r}$ in the first place, if it would produce these consequences. Adding the following condition to the cases in Section 2 suffices: vertex $v_{r}$ on $H$ may not be such that the extension of one of its incident edges hits $p_{r} g_{r}$ unless vertex $g_{r}$ is convex or the extension of the edge clockwise from $g_{r}$ through $g_{r}$ intersects $p_{r} v_{r}$. The addition of this condition does not change the fact that one of the cases will eventually apply, since if the condition does not hold, the extension of the counterclockwise edge from $g_{r}$ hits $H$ and $g_{r}$ cannot serve as $b$. The next step taken by segment $h^{\prime} p^{\prime}$ is to move $h^{\prime}$ to $v_{r}$.

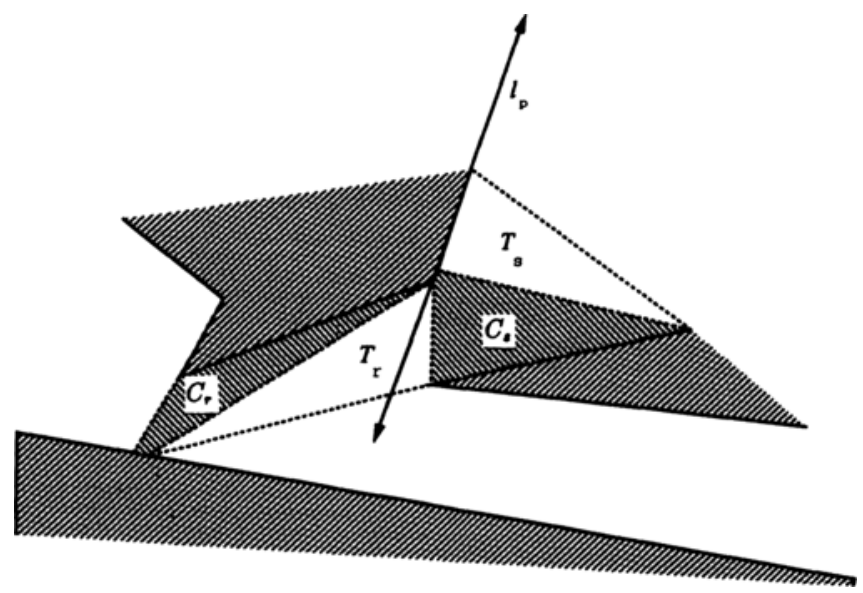

Fig. 23. If $l_{p}$ intersects $t_{2} t_{3}$, then Case 2.2 applies. 


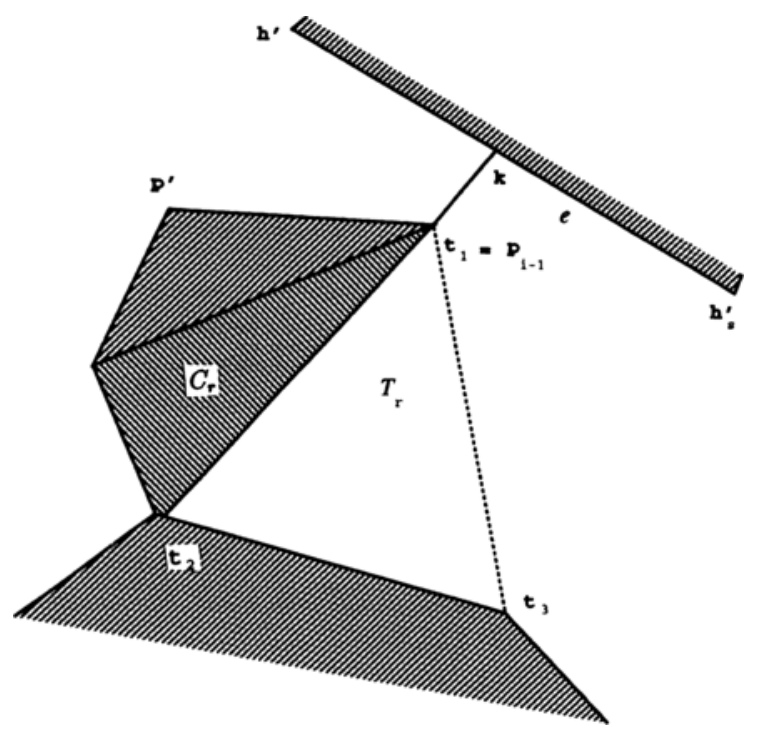

Fig. 24. Extending edge $t_{2} t_{1}$ through $t_{1}$ makes $Q_{r}$ nonempty in $P$.

Instance 3. Vertex $t_{1}$ is moved.

Vertex $t_{1}$ can move if the construction associated with Case 2.3 is used to produce $C_{s}$ with the result that $Q_{r}$ becomes nonempty in $P$ (see Fig. 24). We prevent this behavior by modifying Case 2.3 to include the condition that the extended side $f^{\prime}$ may not be the side of any channel $C_{r}$ supporting an associated triangle $T_{r}$. This restriction does not preclude the existence of an applicable case. An empty convex quadrilateral $Q_{s}$ must exist or else Case 2.3 would not apply. In $Q_{s}$ replace the point $k$ where the extension from $t_{1}$ hits $e$ with the other endpoint $h_{s}^{\prime}$ of $e$. Let the new quadrilateral be $Q_{s}^{\prime}$. If $Q_{s}^{\prime}$ is empty and convex, then Case 2.2 applies. If $Q_{s}^{\prime}$ is nonempty, then vertex $x$ exists such that either $h^{\prime}$ or $p^{\prime}$ next moves to $x$. If $Q_{s}$ is nonconvex and empty, then $p^{\prime}$ moves to $t_{1}$. At this point, extend an edge from $t_{1}$ again, but use the other incident edge. A new vertex $t^{\prime}$ is created next to $t_{1}$ in the direction of $t_{2}$. Let $g_{s}^{\prime}$ be the point where the extension hits $e$. Either quadrilateral $\left(h_{s}^{\prime}, g_{s}^{\prime}, t_{1}, t_{2}\right)$ is convex and empty, or a vertex $v_{s}$ exists such that quadrilateral $\left(h_{s}, g_{s}, t_{1}, v_{s}\right)$ is convex and empty. Thus Case 2.3 holds using this different edge extension. If $t_{2}$ is the vertex chosen for $Q_{s}^{\prime}$, then $Q_{r}^{\prime}=\left(t^{\prime}, h_{r}, p_{r}, g_{s}^{\prime}\right)$ is convex and empty in $P$. The corresponding triangles $T_{r}$ and $T_{s}$ are identical and exist in $P^{s}$. If some other vertex $v_{s}$ is chosen for $Q_{s}^{\prime}$, then $Q_{r}^{\prime}=\left(t^{\prime}, h_{r}, p_{r}, t_{3}\right)$ is convex and empty in $P, t^{\prime}$ replaces $t_{1}$, and $T_{r}$ exists.

Instance 4. Vertex $t_{3}$ is cut off from $T_{r}$ by the intended channel $C_{s}$.

This instance resembles Instance 2 where vertex $t_{3}$ moves onto edge $t_{2} p_{j}$. Channel $C_{s}$ creates two new vertices on side $t_{2} t_{3}$ (or $t_{2} p_{j}$ ). Let these two 


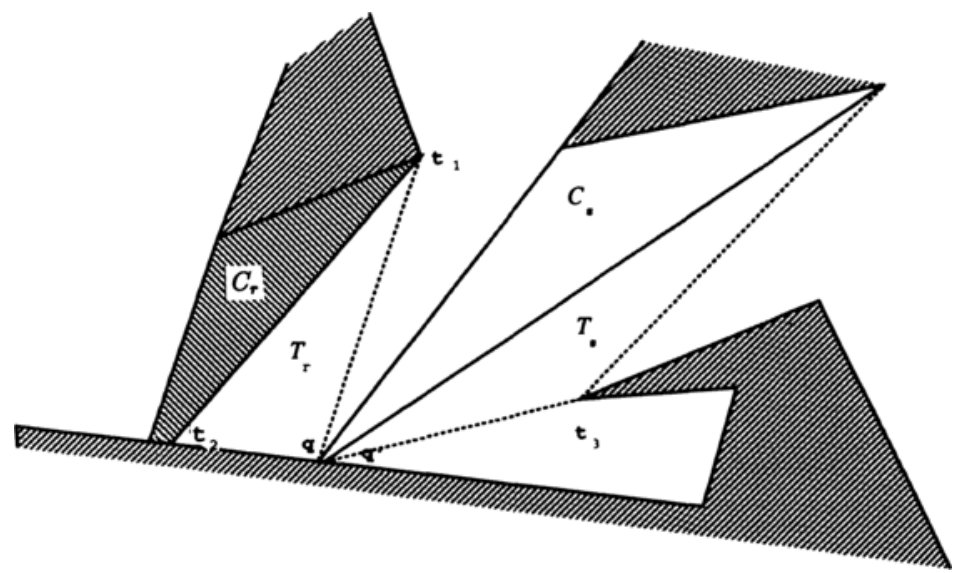

Fig. 25. $T$, shrinks to become triangle $\left(t_{1}, t_{2}, q\right)$.

vertices be $q$ and $q^{\prime}$. If $q$ is closer to $t_{2}$, then $q$ replaces $t_{3}, Q_{r}^{\prime}=\left(t_{1}, h_{r}, p_{r}, q\right)$ is convex and empty in $P$, and $T_{r}$ exists in $P^{s}$ (see Fig. 25). If $q^{\prime}$ is the vertex closer to $t_{2}$, then $T_{r}$ and $T_{s}$ must share the area $t_{1}, t_{2}, q^{\prime}, g_{s}$ (see Fig. 26). $T_{r}$ becomes the triangle $\left(t_{1}, t_{2}, q^{\prime}\right)$ and $T_{s}$ is chosen using vertices $q^{\prime}, g_{s}$, and $t_{x}$ or $T_{r}$ is chosen using $t_{1}, t_{2}$, and $g_{s}$ and $T_{s}$ becomes triangle $\left(g_{s}, t_{2}, q^{\prime}\right)$.

Instance 5. Vertex $t_{2}$ is cut off from $T_{r}$ by the intended channel $C_{s}$.

This instance occurs only if $t_{2} t_{3}$ is interior to $P^{r}$. We attempted to construct channel $C_{s}$ so that its 8 -side lies along edge $t_{1} t_{2}$, but then $T_{r}$ would no longer exist within $P^{s}$. A new vertex must be chosen to replace this $t_{2}$. Let the two vertices of

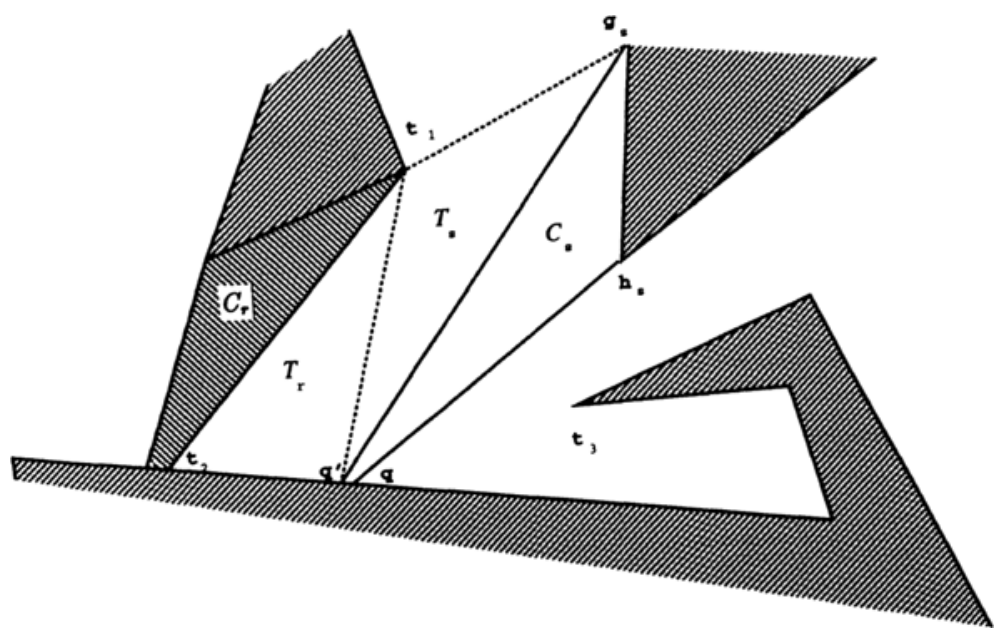

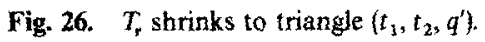




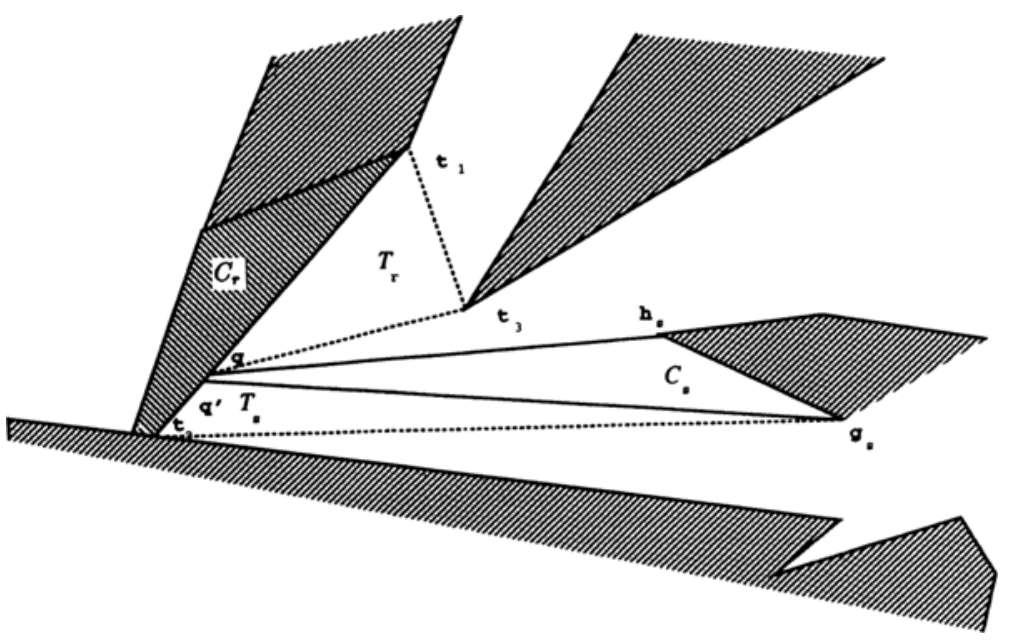

Fig. 27. $T_{r}$ shrinks to triangle $\left(t_{1}, q, t_{3}\right)$.

$C_{s}$ on edge $t_{1} t_{2}$ be $q$ and $q^{\prime}$. Whichever of $q$ or $q^{\prime}$ lies on the same side of $C_{s}$ as $t_{1}$ and $t_{3}$ will replace $t_{2}$ in $T_{r} . T_{r}$ in its modified form still has one side along the side of channel $C_{r}$ and its three vertices, if superimposed on $P_{r}$ (and $P$ ), all see the entire area covered by $C_{r}$. Specifically, if $q$ is closer to $t_{1}, T_{r}$ becomes the triangle $\left(t_{1}, q, t_{3}\right)$ (see Fig. 27). The vertex $v_{s}$ which completes $Q_{s}$ lies on the other side of $C_{s}$, and triangles $T_{r}$ and $T_{s}$ now do not interfere with each other. If $q^{\prime}$ is closer to $t_{1}, T_{r}$ shrinks to triangle $\left(t_{1}, q^{\prime}, \mathrm{t}_{3}\right)$ (see Fig. 28). In this case triangles $T_{r}$ and $T_{s}$ lie on the

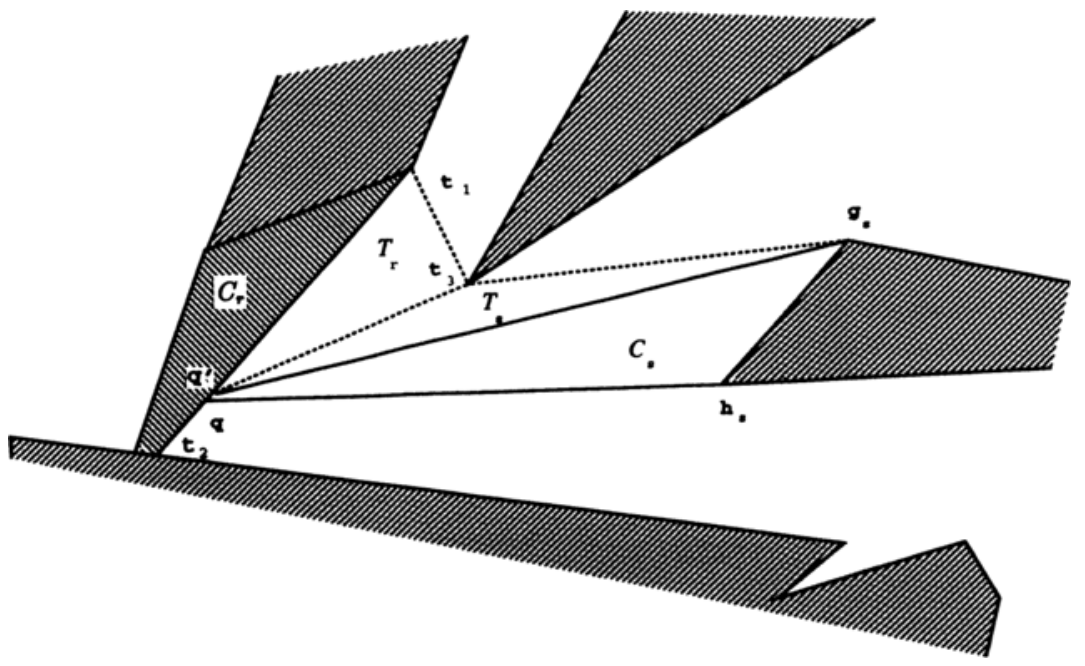

Fig. 28. $T_{\mathrm{r}}$ shrinks to triangle $\left(t_{1}, q^{\prime}, t_{3}\right)$. 
same side of $C_{s}$. By the choice of $t_{3}$ we know that vertex $v_{s}$ which becomes the apex of $T_{s}$ either is identical with the vertex $t_{3}$ or lies inside the quadrilateral $\left(g_{s}, h_{s}, q, t_{3}\right)$. Thus triangles $T_{r}$ and $T_{s}$ do not interfere with each other and both exist in $P^{s}$.

Instance 6. Vertex $t_{1}$ is cut off from $T_{r}$ by the intended channel $C_{s}$.

In this case we wish to place the $\varepsilon$-side of $C_{s}$ along $t_{1} t_{2}$ by using an extension from some vertex $h_{s}$ on a hole. When $C_{r}$ was constructed, the additional condition from Instance 2 above must have held or $C_{r}$ would not have been constructed. We thus know that either some other vertex $t$ that is encountered before $h_{k}$ when $t_{1} t_{2}$ rotates toward the extension through $t_{1}$ (if $t_{2} t_{3}$ is interior to $P$, then $t=t_{3}$ ) exists, or the extension of the edge counterclockwise from $t_{1}$ intersects $t_{2} h_{s}$. In the first case we first replace $t_{3}$ by $t$ in $T_{r}$. If this replacement forces $C_{s}$ to cut off vertex $t_{2}$ instead of $t_{1}$ (see Fig. 29), we continue the modifications as in Instance 5 . If the replacement does not result in Instance 5 , we return to our original $t_{3}$ and let $q$ and $q^{\prime}$ be the two new vertices on $t_{1} t_{2}$. With $q^{\prime}$ closer to $t_{1}, T_{r}$ shrinks to the triangle $\left(q, t_{2}, t_{3}\right)$ (see Fig. 30) and $T_{s}$ is chosen normally using $q^{\prime}, t_{1}$, and $g_{s}$. The modified $T_{r}$ exists in $P^{s}$, and, since the vertex replacing $t_{1}$ lies on the side $t_{1} t_{2}$, every vertex of $T_{r}$ still sees all of the area covered by $C_{r}$ in $P$. If $q$ is closer to $t_{1}, T_{r}$ becomes the triangle $\left(q^{\prime}, t_{2}, t_{3}\right)$ (see Fig. 31). To make sure that $T_{r}$ and $T_{s}$ both exist, we look at the convex quadrilateral $Q_{s}=\left(g_{s}, h_{s}, q^{\prime}, t_{3}\right)$. If $Q_{s}$ is empty, then $T_{s}$ is the triangle $\left(g_{s}, q^{\prime}, t_{3}\right)$; if $Q_{s}$ is nonempty, then one of the vertices in $Q_{s}$ becomes the third vertex of $T_{s}$. In either case both $T_{r}$ and $T_{s}$ exist in $P^{s}$. Finally, if the edge counterclockwise from $t_{1}$ through $t_{1}$ intersects $t_{2} h_{s}$, we know that unless vertex $h_{s}$ is the vertex where we actually start the search for a channel, Case 2.2 would have applied at an earlier step just as in Instance 2 . Consequently,

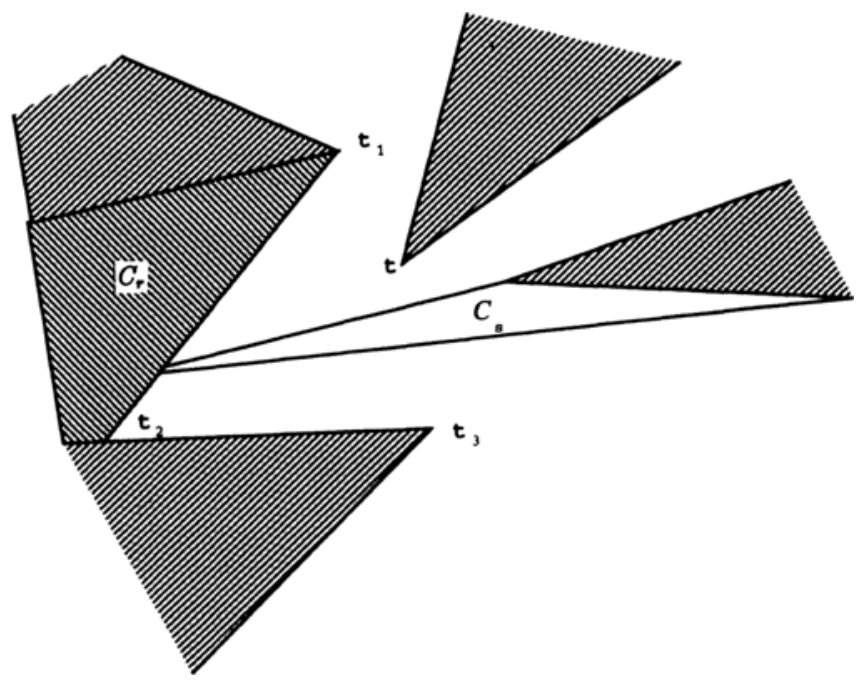

Fig. 29. When $t$ replaces $t_{3}$ in $T_{r}$, channel $C_{s}$ cuts off $t_{2}$ instead of $t_{1}$ from $T_{r}$. 


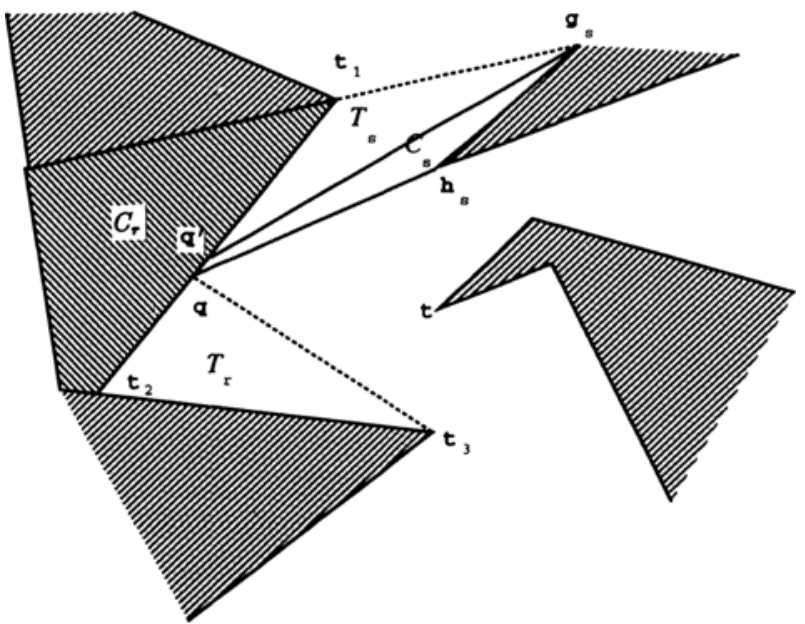

Fig. 30. $T_{r}$ shrinks to triangle $\left(q, t_{2}, t_{3}\right)$.

$h_{s}=a$ and we attempt to apply the construction of Case 2.1 to create channel $C_{s}$. If $l_{s}$ and an extension of $t_{2} t_{3}$ through $t_{3}$ intersect and the area in between is empty, we move $p^{\prime}$ to $t_{2}$ and continue the search. Otherwise, we simply go ahead and construct the intended channel as above with $q$ closer to $t_{1}$.

As demonstrated above, a triangle $T_{r}$ may go through several modifications before all channels have been constructed (see Fig. 32), but the revised versions of Cases 2.1-2.3 guarantee that a satisfactory $T_{r}$ will exist in $P^{h}$ :

Case 3.1. A reflex vertex $h_{k}$ on $H$ with incident edges $e=h_{k} h_{l}$ and $e^{\prime}$ and an edge $f=p_{i} p_{j}$ on $B$ exists such that the following conditions hold:

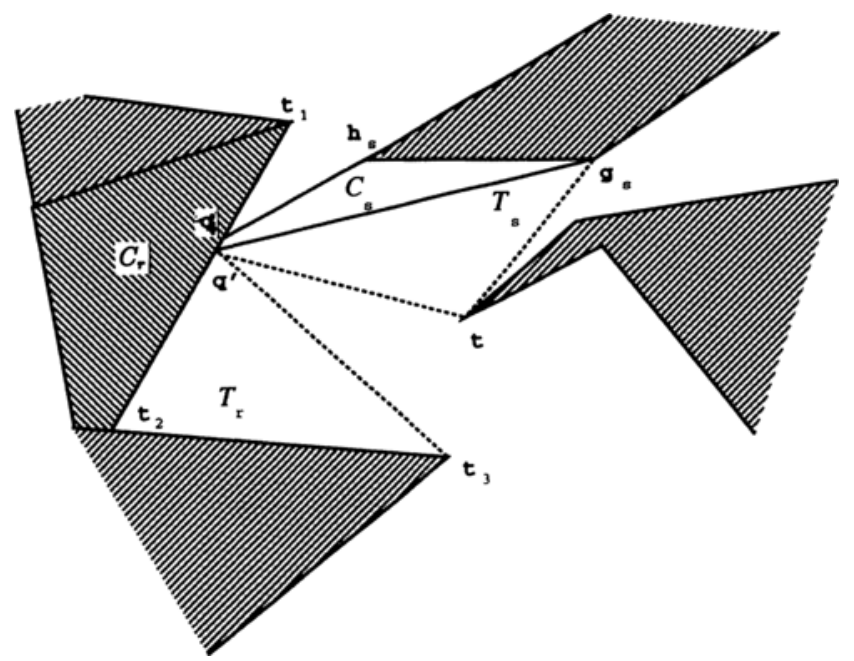

Fig. 31. $T_{r}$ shrinks to triangle $\left(q^{\prime}, t_{2}, t_{3}\right)$. 

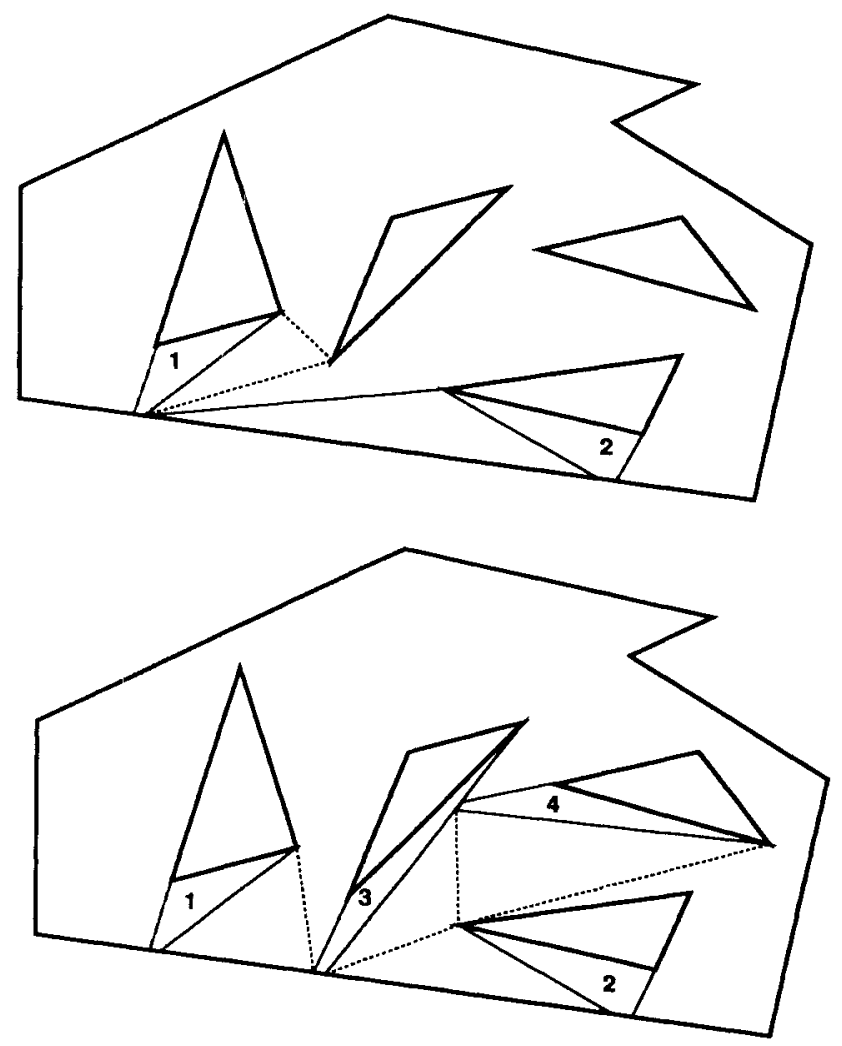

Fig. 32. The channels are constructed in numerical order.

- The extension of $e^{\prime}$ through $h_{k}$ hits $f$ at $q \neq p_{j}$.

- A half-plane defined by $\overline{h_{k} q}$ contains both $e$ and $p_{j}$.

- A vertex $v$ on $B$ or $H$ and a quadrilateral $Q=\left(h_{l}, h_{k}, q, v\right)$ such that:

1. $v \notin \overline{q h_{l}}$.

2. $v$ is the first vertex encountered by $h_{l} q$ as $q$ slides along $f$.

3. $v=p_{j} \Rightarrow P \cup$ triangle $\left(h_{l}, v, z\right) \neq\left\{h_{l}, v, z\right\}$.

4. $Q$ is convex.

5. $Q$ has an empty interior.

- $f$ is not the base of a triangle $T$ with $h_{k}$ designated $t_{3}$.

- There is no vertex $u$ on $H$ such that:

1. Excluding $p_{j}, u$ is the first vertex encountered by $h_{l} q$ as $q$ slides along a line through $f$.

2. The extension of an edge incident with $u$ intersects $h_{l} q$.

3. $u, h_{l}, h_{m}$ is a left turn, where $h_{m}$ is the endpoint of edge $e^{\prime \prime} \neq e$ incident with $h_{l}$. 
Case 3.2. An edge $e=h_{k} h_{l}$ on $H$ and an edge $f=p_{i} p_{j}$ on $B$ exists such that the following conditions hold:

- A half-plane defined by $\overline{h_{k} p_{i}}$ contains both $e$ and $p_{j}$.

- A vertex $v$ on $H$ or $B$ and a quadrilateral $Q=\left(h_{l}, h_{k}, p_{i}, v\right)$ such that:

1. $v \notin \overline{p_{i} h_{l}}$.

2. $v$ is the first vertex encountered by $h_{l} p_{i}$ as $p_{i}$ slides along $f$.

3. $v=p_{j} \Rightarrow P \cup$ triangle $\left(h_{l}, v, z\right) \neq\left\{h_{t}, v, z\right\}$.

4. $Q$ is convex.

5. $Q$ has an empty interior.

- There is no vertex $u$ on $H$ such that:

1. Excluding $p_{j}, u$ is the first vertex encountered by $h_{i} p_{i}$ as $p_{i}$ slides along a line through $f$.

2. The extension of an edge incident to $u$ intersects $h_{l} p_{i}$.

3. $u, h_{l}, h_{m}$ is a left turn, where $h_{m}$ is the endpoint of edge $e^{\prime \prime} \neq e$ incident with $h_{l}$.

Case 3.3. An edge $e=h_{k} h_{l}$ on $H$ and a reflex vertex $p_{i}$ with incident edges $f$ and $f^{\prime}$ on $B$ exist such that the following conditions hold:

- The extension of $f^{\prime}$ through $p_{i}$ hits $e$ at $k \neq h_{l}$.

- A half-plane defined by $\overline{p_{i} k}$ contains both $f$ and $h_{l}$.

- A vertex $v$ on $H$ or $B$ and a quadrilateral $Q=\left(h_{l}, k, p_{i}, v\right)$ exist such that:

1. $v \notin \overline{p_{i} h_{l}}$.

2. $v$ is the first vertex encountered by $h_{l} p_{i}$ as $p_{i}$ slides along $f$.

3. $v=p_{j} \Rightarrow P \cup$ triangle $\left(h_{l}, v, z\right) \neq\left\{h_{l}, v, z\right\}$.

4. $Q$ is convex.

5. $Q$ has an empty interior.

- $f^{\prime}$ is not the base of a triangle with $p_{i}$ designated $t_{1}$.

- There is no vertex $u$ on $H$ such that:

1. Excluding $p_{j}, u$ is the first vertex encountered by $h_{l} p_{i}$ as $p_{i}$ slides along a line through $f$.

2. The extension of an edge incident to $u$ intersects $h_{l} p_{i}$.

3. $u, h_{l} h_{m}$ is a left turn, where $h_{m}$ is the endpoint of edge $e^{\prime \prime} \neq e$ incident with $h_{l}$.

When $h$ channels have been constructed, every part of each channel is visible from a triangle and the interiors of these triangles are all disjoint. In addition, the construction of the channels results in a simple polygon $P^{h}$ with at most $n+h$ vertices and no holes. The triangles $T_{1}, \ldots, T_{h}$ are inside this polygon. We remove the triangles and triangulate the remaining parts. Putting the pieces together again we have a triangulated polygon in which, after three-coloring, we can place $\lfloor(n+h) / 3\rfloor$ vertex guards that see all of $P^{h}$ (see Fig. 33). Since each of the triangles $T_{1}, \ldots, T_{h}$ has a guard at at least one vertex, these guards see all of the 

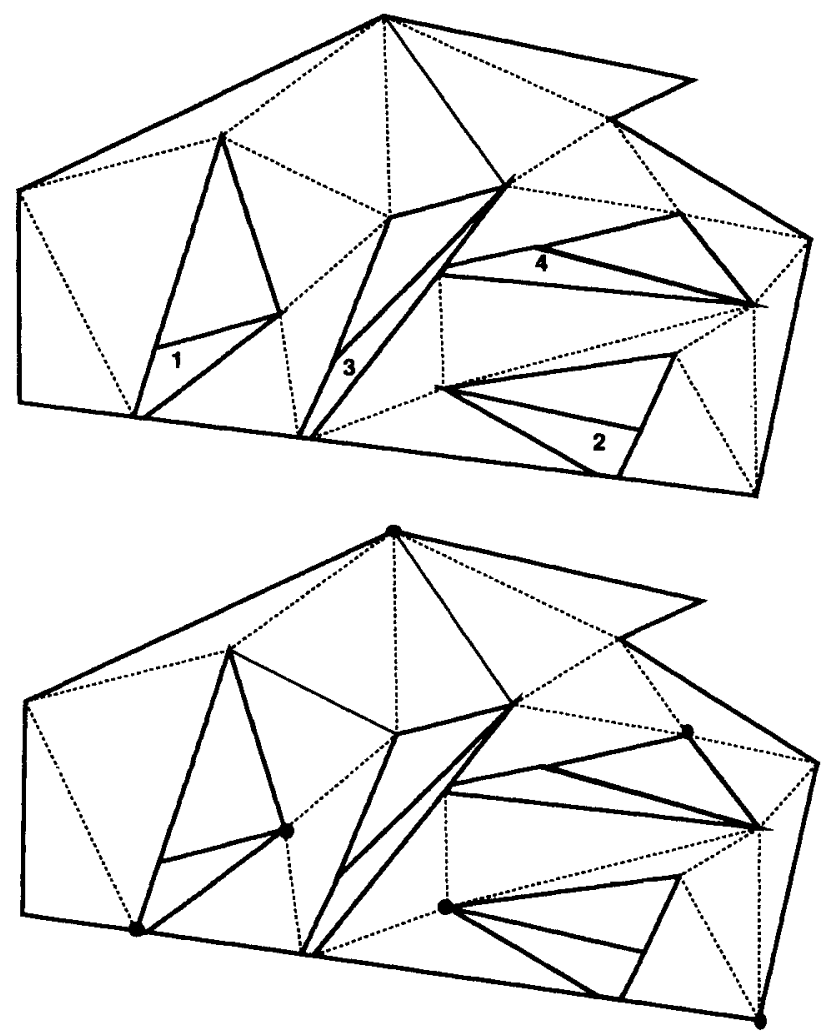

Fig. 33. The hole-free polygon is triangulated and guards placed based on three-coloring. These guards see all of the original polygon.

original polygon $P$. The guards are point guards, since $P^{h}$ has vertices not present in $P$. This proves the following theorem:

Theorem 3.1. $\lfloor(n+h) / 3\rfloor$ point guards are sufficient to cover the interior of a simple polygon of $n$ vertices and with $h$ holes.

\section{Algorithm for Placing the Guards}

We use the following argument to show that $O\left(n^{2}\right)$ time will suffice to turn the polygon $P$ into a simple polygon $P^{h}$ with no holes and $n+h$ vertices: for each pair of vertices $h^{\prime}$ and $p^{\prime}, O(n)$ time suffices to check whether a channel between the edges $e$ and $f$ is possible; during the algorithm, at most $O(n)$ edge pairs are checked. Although the triangulation, three-coloring, and placing of the guards within $P^{h}$ can be done in linear time, if Chazelle's triangulation algorithm is used, we use the practical algorithm by Avis and Toussaint, which runs in $O(n \log n)$ time. Thus the overall time bound is $O\left(n^{2}\right)$. 
Consider the case of one hole. Let $H$ be the hole and let $B$ be the exterior boundary of the polygon. Following the constructive proof of the theorem in Section 2, we start by finding a vertex $a$ on $H$, to serve as $h^{\prime}$. We next find the edge first intersected by the extension of the edge clockwise from $a$ through $a$ and compute $r=p^{\prime}$. Given the segment $h^{\prime} p^{\prime}$, we try each channel construction in turn, if none is possible either $h^{\prime}$ or $p^{\prime}$ is moved one step in a counterclockwise direction and we try again. All variable names are those used in Sections 2 and 3.

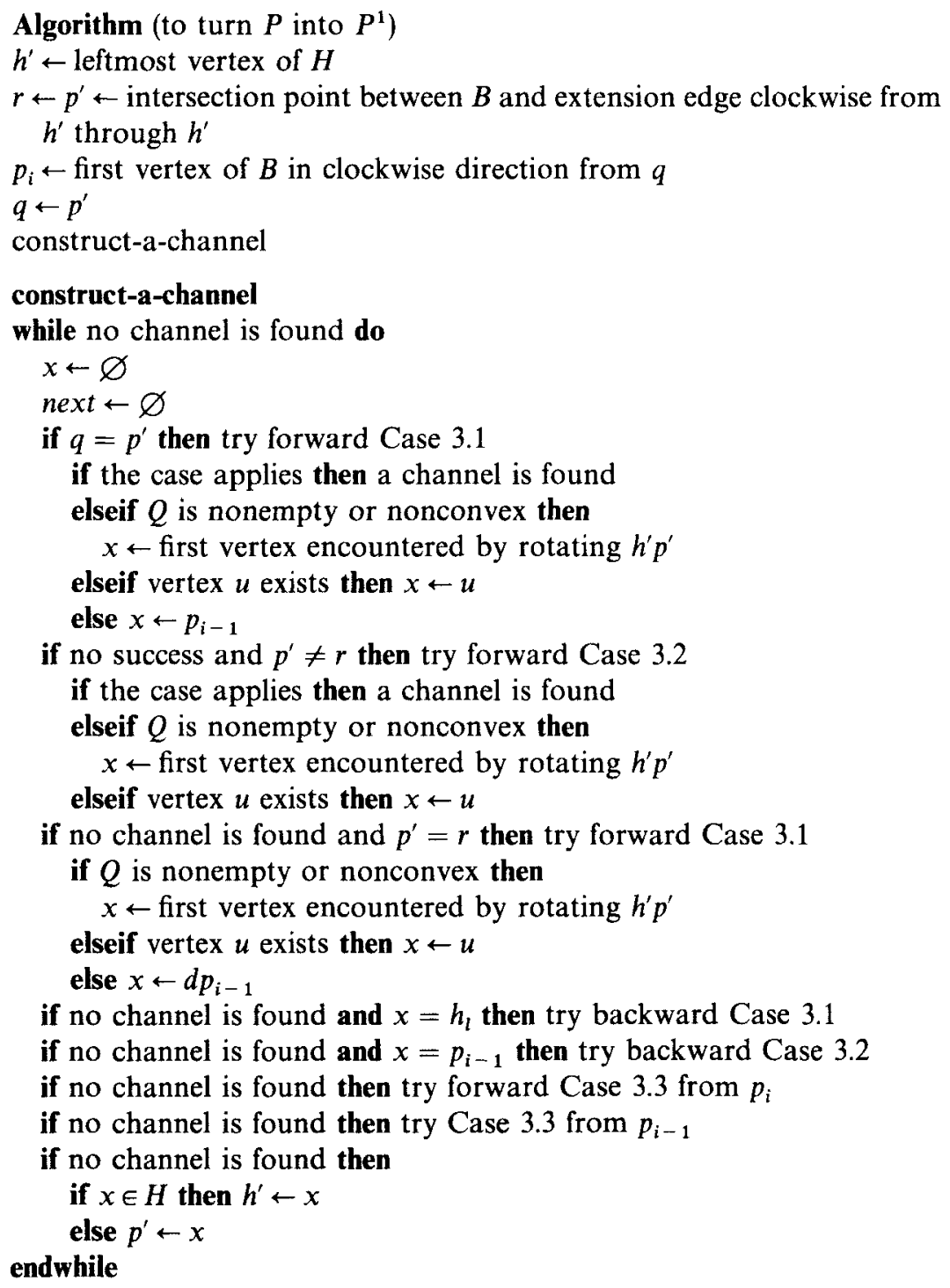

Given a tentative pair $h^{\prime}$ and $p^{\prime}$, it takes linear time to try one construction. This is so, since all that is involved in an attempted construction is finding the 
intersections between two lines and checking whether any vertex lies inside the intended channel or the associated triangle. This can be done in linear time. Since only a constant number of constructions are tried, the while loop in the constructa-channel algorithm runs in linear time. Once replaced, a particular vertex never again becomes the current $h^{\prime}$ or $p^{\prime}$. Thus each time through the while loop either $h^{\prime}$ or $p^{\prime}$ must be previously unseen. Therefore, we run through the while loop at most $n$ times and the total time taken by construct-a-channel is $O\left(n^{2}\right)$. Picking an initial $h^{\prime}$ and finding the corresponding $p^{\prime}$ and $p_{i}$ is also done in linear time. Thus the complete algorithm for one hole takes $O\left(n^{2}\right)$ time.

When there is more than one hole, the search for channels does not stop until all holes are connected to the exterior. Keeping $P$ and $H_{1}, \ldots, H_{h}$ as doubly linked lists enables us to update the polygon $P$ in constant time, since when we are considering a particular pair $h^{\prime}$ and $p^{\prime}$, we are at the correct point in the lists to adjust pointers and delete and add appropriate vertices. Each time a channel is constructed, the search for the next channel continues in such a way that all but one of the vertex pairs producing a candidate channel contains a previously unseen vertex. To ensure this phenomenon, throughout the search, we record each place where the search jumps from one hole to another, by pushing the pair old $h^{\prime}$, old $p^{\prime}$ on a stack $S$. After a channel has been constructed, pop the stack and check if the indicated hole has been connected. If so, pop the stack again; else continue the search from the popped vertices. The vertex which last time became the new $h^{\prime}$ now lies on the exterior boundary and becomes the new $p^{\prime}$. The search continues from there along a new route.

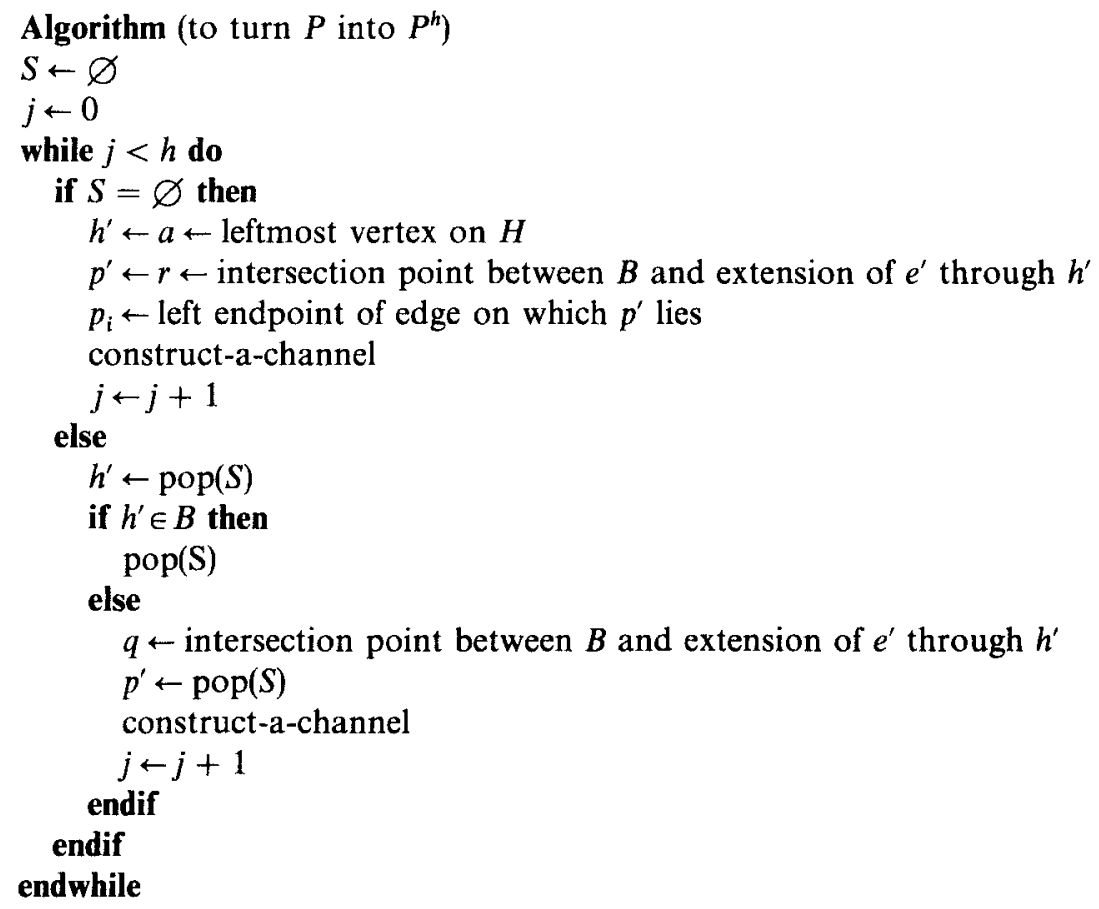


Either at the start the search or at any intermediary point when the stack is empty and holes still exist, we pick the leftmost hole vertex and the point where an extension of the edge clockwise from this vertex intersects the boundary as the place to continue the search. When the stack is empty, all vertices previously considered now lie on the current exterior boundary. Thus this initial pair will contain a previously unseen vertex. If no channel construction is found for this pair and $p^{\prime}$ is replaced by an obstructing vertex, this vertex may have been previously seen, but this can occur at most once each time we restart the search, i.e., at most once for each hole. In summary, since the number of holes is $h$, we investigate at most $O(n+h)$ edge pairs for possible channel constructions. Spending $O(n)$ time on each edge pair gives us a total time of $O\left(n^{2}\right)$.

\section{Conclusions}

In this paper we studied the problem of assigning stationary guards in polygons with holes. We showed a bound for the number of guards required and gave an algorithm for placing the guards that runs in $O\left(n^{2}\right)$ time, where $n$ is the total number of vertices. The algorithm was derived from the constructive proof of the upper bound.

We note that a new polygon $P^{h}$ without holes was constructed from the original polygon $P$ in such a way that several new vertices were created. Therefore, although the chosen guards are vertex guards in $P^{h}$, these vertices need not exist in $P$ and the same guards are instead point guards in the original polygon. The problem of finding a tight bound on the number of vertex guards required to guard a polygon with $h$ holes and a total of $n$ vertices remains open. $\lfloor(n+h) / 3\rfloor$ vertex guards are certainly necessary but the best-known upper bound is $\lfloor(n+2 h) / 3\rfloor$ established by O'Rourke [8] in 1982.

\section{Acknowledgments}

We thank Joseph O'Rourke for the many helpful comments and suggestions to improve the presentation of this paper. The paper has also benefited from the comments of an anonymous referee.

\section{References}

1. D. Avis and G. T. Toussaint, An efficient algorithm for decomposing a polygon into star-shaped pieces, Pattern Recognition 13 (1981), 395-398.

2. I. Bjorling-Sachs and D. Souvaine, A Tight Bound for Guarding Polygons with Holes, Rutgers University Technical Report LCSR-TR-165, May 1991. Abstract appears in Final Report of MSI Stony Brook Workshop on Computational Geometry, Stony Brook, Oct. 1991, p. 17.

3. V. Chvátal, A combinatorial theorem in plane geometry, Journal of Combinatorial Theory, Series B 18 (1975), 39-41. 
4. S. Fisk, A short proof of Chvátal's watchman theorem, Journal of Combinatorial Theory, Series B 24 (1978), 374.

5. F. Hoffmann, Colloquium, Department of Computer Science, Rutgers University, 24 Oct. 1991.

6. F. Hoffmann, M. Kaufmann, and K. Kriegel, The art gallery theorem for polygons with holes, Proceedings of the Symposium of Foundations of Computer Science, 1991, pp. 39-48.

7. R. Honsberger, Mathematical Gems II, Mathematical Association of America, Washington, DC, 1976, pp. 104-110.

8. J. O'Rourke, Art Gallery Problems and Algorithms, Oxford University Press, Oxford, 1987.

9. G. T. Toussaint, An optimal algorithm for computing the relative convex hull of a set of points in a polygon, Signal Processing III: Theories and Applications (Proceedings of the Third European Signal Processing Conference) (1986), pp. 853-856.

Received March 30, 1993, and in revised form June 7, 1994. 